\title{
LA-5061-MS
}

$40.235^{\circ}$

$11-28^{\circ}>2$

245

INFORMAL REPORT

\section{A Monte Carlo Sampler}

\section{ociontifie laboratory}

of the University of California

iOS alamos, NEW MEXICO 87544

1 
This report was prepared as an account of work sponsored by the United States Government. Neither the United States mor the United States Atomic Energy Commission, nor ariy of their employees, nor any of their contractors, subcontractors, or their employees, makes any warranty, express or implied, or assumes any legal liability' or responsibility for the accuracy, completeness or usefulness of any information, apparatus, product or process disclosed, or represents that its use would not infringe privacely owned rights.

In the interest of prompt distribution, this LAMS report was not edited by the Technical Information staff.

Printed in the United States of America. Available fiom National Technical Information Service

U. S. Department of Commerce 5285 Port Royal Road Springfield, Virginia 22151

Price: Printed Copy \$3.00; Microfiche \$0.95 
LA-5061-MS

Informal Report

UC-32 and 34

ISSUED: October 1972
This report was prepared ICE

sponsored by the prepared as an account of work

the United States nor the States Government. Neither

Commission, nor any of United States Atomic Energy

their contraciors, subor their employees, nor any of

makos any warranty, oxprostors,

legal liability or ly, axpress or implied, or assumes any

pleteness or ur es accuracy, com-

product or process dis of any information, apparatus.

would not infringe privately, or represents that its use

\section{A Monte Carlo Sampler}

by

C. ¿. Everett

E. D. Cashwell 


\title{
A MONTE CARLO SAMPLER
}

by

\author{
C. J. Everett and E. D. Cashwell
}

\begin{abstract}
Methods are given for sanpling the standard probability densities arising in physical, chemical, and statisticial problems, by means of machine generated "random numberis." The probability theory underlying each device is briefly indlcated. The collection is intended as a reference work for Monte Carlo practice. No attempt is made to cuote original sources, and no claim to priority is intencled in any case.
\end{abstract}

FOREWORD

In $a 11$ cases, the: density to ta sampled is followed by a rule ( $R$ ) for choice of the variabie, in terms of random numbers $r_{0}, r_{1}, \ldots$, uniform on $(0,1)$. A justification ( $J$ ) for the rule is usually given, frequently supported by various formulas $(F)$. The indices $D, C, R$ provide "key words" which may help in locating a desired density, but details in this direction are omitted.

\section{FORMULAS}

$$
\begin{aligned}
& \text { F1. } \frac{d}{d u} \int_{g(u)}^{h(u)} f(u, v) d u= \\
& h^{-}(u) f(u, h(u))-g-(u) f(u, g(u))+\int_{g(u)}^{h(u)} \frac{\partial}{\partial u} f(u, v) d v \\
& \text { F2. } \frac{d}{d u} \int_{\left\{v_{1}+v_{2}\left\langle u, v_{1}>0\right\}\right.} p_{1}\left(v_{1}\right) d v_{1} p_{2}\left(v_{2}\right) d v_{2}=\frac{d}{d u} \int_{0}^{u} p_{1}\left(v_{1}\right) d v_{1} \int_{0}^{u-v_{1}} p_{2}\left(v_{2}\right) d v_{2}
\end{aligned}
$$




$$
\begin{gathered}
\equiv \frac{d}{d u} \int_{0}^{u} f\left(u, v_{1}\right) d v_{1}=f(u, u)+\int_{0}^{u} \frac{\partial}{\partial u} f(u, u) d v_{1}=0+\int_{0}^{u} p_{1}\left(v_{1}\right) d v_{1} p_{2}\left(u-v_{1}\right) \\
\text { F3. } \int_{0}^{v} v^{n-1} e^{-b v_{d}} d v=(n-1) 1 b^{-n}\left\{1-e^{-b u} \sum_{0}^{n-1}(b v)^{i} / i t\right\} ;
\end{gathered}
$$$$
0<v<\infty, b>0, n=1,2,3, \ldots
$$$$
D_{b} \equiv \int_{1}^{\infty} v^{n-1} e^{-b v} d v=(n-1) ! b^{-n} e^{-b} s_{b}, s_{b} \equiv \sum_{0}^{n-1} b^{i} / 11 .
$$$$
\text { F4. } \quad r(n) \equiv \int_{0}^{\infty} u^{n-1} e^{-u} d u=2 \int_{0}^{\infty} v^{2 n-1} e^{-v^{2}} d v ; n \text { real }>0 \text {. }
$$$$
\Gamma\left(\frac{1}{2}\right)=\sqrt{\pi}, \Gamma(n+1)=n \Gamma(n), 2^{n-l} \Gamma\left(\frac{n}{2}\right) \Gamma\left(\frac{n+1}{2}\right)=\Gamma\left(\frac{1}{2}\right) \Gamma(n) .
$$

For $n=0,1,2, \ldots, r(n+1)=n !, 01 \equiv 1$.

F5. $B(m, n) \equiv \int_{0}^{1} v^{m-1}(1-v)^{n-1} d v=2 \int_{0}^{\pi / 2} \sin ^{2 m-1} \theta \cos ^{2 n-1} \theta d \theta$

$=I(m) \Gamma(n) / \Gamma(m+n) ; \quad m, n$ real $>0$.

$$
\text { F6. } \int_{\left\{\Sigma_{1}^{n} \mu_{i}<1, \mu_{i}>0\right\}} \pi_{i}^{s i-1} d \mu_{i}=\pi_{1}^{n} \Gamma\left(s_{i}\right) / \Gamma\left(1+\sum_{i}^{n} s_{i}\right) ; s_{i} \text { real >0. }
$$




$$
\text { F7. } v(u)=\int_{\left\{\Sigma_{1}^{n} v_{1}<u, v_{1}>0\right\}}^{n} \pi_{1}^{n} d v_{1}=u^{n} / n 1 \text {, }
$$

$$
d V / d u=u^{n-1} /(n-1) t=A(u)
$$

$$
\begin{aligned}
& \text { F8. } v(u)=\int \Pi_{1}^{N} d v_{1}=\pi^{N / 2} u^{N / 2} 2^{N-1} N \Gamma(N / 2) \text {, } \\
& \left\{\left(\Sigma_{1}^{n} v_{i}^{2}\right)^{\frac{1}{2}}<u, v_{i}>0\right\}
\end{aligned}
$$

$d V / d u=\pi^{N / 2} u^{N-1} / 2^{N-1} \Gamma(N / 2)=A(u)$

Note. $V(u)=2 \pi^{N / 2} u^{N} / N \Gamma(N / 2)$ is the volume of the full sphere of radius $u$, $A(u)=2 \pi^{N / 2} u^{N-1} / \Gamma(N / 2)$ its area. Area of unit sphere is $A(1)$ $=2 \pi^{N / 2} / \Gamma(N / 2)=\int_{\Omega} d \Omega$, where $\Omega$ is the direction vector in $N$-space.

F9. $\zeta(n) \equiv \sum_{1}^{\infty} j^{-n} ; n$ real $>1$.

$$
\begin{aligned}
& \zeta_{a}(n) \equiv \sum_{1}^{\infty}(-1)^{j+1} j^{-n}=\left(1-1 / 2^{n-1}\right) \zeta(n) \\
& \zeta_{u}(n) \equiv \sum_{1}^{\infty}(2 j-1)^{-n}=\left(1-1 / 2^{n}\right) \zeta(n) \\
& \zeta(2 n)=(-1)^{n-1}(2 \pi)^{2 n} B_{2 n} / 2(2 n) !, n=1,2,3 \ldots
\end{aligned}
$$
$B_{2}=1 / 6$
$B_{4}=-1 / 30$
$B_{6}=1 / 42 \quad$ (Bernoulli numbers).
$r(2)=\pi^{2} / 6$
$\zeta(4)=\pi^{4} / 90$
$\zeta(6)=\pi^{6} / 945$
$\zeta(3)=1.2021 \ldots$ 
F10. $\sum_{i}^{n} F_{i}=0$, where

$F_{i} \equiv 1 /\left(a_{1}-a_{i}\right) \cdots\left(a_{i-1}-a_{i}\right) \cdot\left(a_{i+1}-a_{i}\right) \cdots\left(a_{n}-a_{i}\right), n>2$.

Proof. For $f(z) \equiv 1 /\left(a_{1}-z\right) \cdots\left(a_{n}-z\right)$, one has

$(1 / 2 \pi i) \int_{C} f(z) d z=\sum_{1}^{n} \operatorname{Res}\left(a_{j}\right)$, where $C$ is any circle of radius $R>\max \left|a_{i}\right|$, and -Res $\left(a_{i}\right)=F_{i}$, as defined. But $\left|\int_{C} f(Z) d Z\right|<2 \pi R \max _{C}|f(Z)|+0$. as $R \rightarrow \infty \quad(n>2)$

F11. $\int_{0}^{\infty} v^{n-1} d v /\left(\Lambda^{-1} e^{v}+1\right)=\sum_{1}^{\infty}(-1)^{j+1}\left(\Lambda^{j} / j^{n}\right) \int_{0}^{\infty} j^{n} v^{n-1} e^{-j v} d v$

$=\zeta_{a}(\Lambda, n) \Gamma(n)$, where $\zeta_{a}(\Lambda, n) \equiv \sum_{1}^{\infty}(-1)^{j+1} \Lambda^{j} / j^{n}, 0<\Lambda<1, n>1$.

F12. $\int_{0}^{\infty} v^{n-1} d v \Lambda e^{-v} /\left(1-\Lambda^{2} e^{-2 v}\right)=$

$\sum_{1}^{\infty}\left(\Lambda^{2 j-1} /(2 j-1)^{n}\right) \int_{0}^{\infty}(2 j-1)^{n} v^{n-1} e^{-(2 j-1) \nu} d v=\zeta_{u}(\Lambda, n) \Gamma(n)$.

were $\zeta_{u}(\Lambda, n) \equiv \sum_{1}^{\infty} \Lambda^{2 j-1} /(2 j-1)^{n}, 0<\Lambda<1, n>1$. 
F13. $K_{N}(u) \equiv \int_{0}^{\infty} \cosh N \theta e^{-u \cosh \theta} d \theta ;(0, \infty), N>0$

(a) $K_{N}(u)=1 / 2 \int_{-\infty}^{\infty} e^{-N \theta} e^{-u \cosh \theta} d \theta($ trivial)

(b) $k_{N}(u)=2^{-(N+1)} u^{N} \int_{0}^{\infty} x^{-(N+1)} e^{-\left(x+\frac{u^{2}}{4 x}\right)} d x\left(e^{\theta}=2 x / u\right)$

(c) $k_{N}(u)=\frac{\Gamma(1 / 2) u^{N}}{2^{N} \Gamma(N+1 / 2)} \int_{1}^{\infty}\left(v^{2}-\Sigma\right)^{N-1 / 2} e^{-u v} d v \quad$ (cf. [12]) $=\frac{\Gamma(1 / 2) u^{N}}{2^{N} \Gamma(N+1 / 2)} \int_{0}^{1} x^{-(2 N+1)}\left(1-x^{2}\right)^{N-\frac{1}{2}} e^{-u / x} d x\left(v=x^{-1}\right)$

(d) $k_{N}\left(2 v^{\frac{1}{2}}\right)=2^{-1} v^{N / 2} \int_{0}^{\infty} x^{-(N+1)} e^{-\left(x+\frac{v}{x}\right)} d x \quad$ (from (b))

F14. $\int_{0}^{\infty} x^{n-1} e^{-b \sqrt{x^{2}+1}} d x=\int_{1}^{\infty} e^{-b v} v\left(v^{2}-1\right)^{\frac{n}{2}-1} d v$ $\equiv \int_{1}^{\infty} u d v=\frac{b}{n} \int_{1}^{\infty}\left(v^{2}-1\right)^{\frac{n}{2}} e^{-b v} d v$

$=\left(\Gamma(n / 2) / \Gamma\left(\frac{1}{2}\right)\right)(2 / b)^{\frac{n-1}{2}} K_{\frac{n+1}{2}}(b) ; n, b>0 \quad$ (cf. $\left.F 13(c)\right)$ 
F15. $\int_{0}^{\infty} x^{n-1} d x /\left(\Lambda^{-1} e^{a \cdot \sqrt{x^{2}+1}}+1\right)$

$$
\begin{aligned}
& =\sum_{1}^{\infty}(-1)^{j+1} \Lambda^{j} \int_{0}^{\infty} x^{n-1} e^{-j a \sqrt{x^{2}+1}} d x \\
& =2^{\frac{n-1}{2}}\left(\Gamma(n / 2) / \Gamma\left(\frac{k}{2}\right)\right) \sum_{1}^{\infty}(-1)^{j+1} \Lambda^{j} \frac{k_{n+1}}{2}(j a) /(j a)^{\frac{n-1}{2}} ; n, a>0 . \quad \text { (cf. F14) }
\end{aligned}
$$

F16. $\int_{1}^{\infty} v^{n-1} \Lambda e^{-a v} /\left(1-\Lambda^{2} e^{-2 a v}\right)=\sum_{1}^{\infty} \Lambda^{2 j-1} \int_{1}^{\infty} v^{n-1} e^{-(2 j-1) a v} d v$

$$
=\sum_{I}^{\infty} \Lambda^{2 j-1} D_{(2 j-1) a j} D_{b} \text { defined as in F3. }
$$

F17. $\int_{0}^{\infty} u^{n-1} K_{N}(u) d u=$

$$
\begin{aligned}
& \left(r\left(\frac{k_{2}}{2}\right) / 2^{N} r\left(N+\frac{1}{2}\right)\right) \int_{1}^{\infty}\left(v^{2}-1\right)^{N-\frac{1}{2}} d v \int_{0}^{\infty} u^{n+N-1} e^{-u N} d u \\
& =\left(r\left(\frac{1}{2}\right) r(n+N) / 2^{N} r\left(N+\frac{k_{2}}{2}\right)\right) \int_{1}^{\infty} v^{-(n+N)}\left(v^{2}-1\right)^{N-\frac{1}{2}} d v
\end{aligned}
$$

6 
$=\left(r\left(\xi_{\xi}\right) \Gamma(n+N) / 2^{N+1} \Gamma\left(N+\frac{\xi_{\xi}}{\xi}\right)\right) \int_{0}^{1} \xi^{\frac{n-N}{2}-1}(1-\xi)^{\left(N+\xi_{2}\right)-1} d \xi$

$=\left(n\left(\frac{k}{2}\right) \Gamma(n+N) / 2^{N+1} \Gamma\left(N+\frac{1}{2}\right)\right) B\left(\frac{n-N}{2} \cdot N+\frac{1}{2}\right)$

$=\Gamma\left(\frac{1}{2}\right) \Gamma(n+N) \Gamma\left(\frac{n-N}{2}\right) / 2^{N+1} \Gamma\left(\frac{n+N+1}{2}\right)$

$=2^{n-2} r\left(\frac{n-N}{2}\right) r\left(\frac{n+N}{2}\right) ; 0<N<n$.

F18. $E_{N}(u) \equiv \int_{1}^{\infty} v^{-N} e^{-u v} d v=\int_{0}^{1} x^{N-2} e^{-u / x} d x_{i}$

$0<u<\infty, n>0$. Cf. [1].

F19. $\int_{0}^{\infty} u^{n-1} E_{N}(u) d u=\int_{1}^{\infty} v^{-N} d v \int_{0}^{\infty} u^{n-1} e^{-u v} d v$

$=I(n) \int_{1}^{\infty} v^{-(n+N)} d v=\Gamma(n) /(n+N-1) ; N>0, n>0, n+N>1$.

\section{D-INDEX \\ Discrete Densities}

D1. $p(v) ; v=0,1,2, \ldots$

D2. $e^{-a} a^{v} / v !$

D3. $P\{f(v)=u\}$

D4. $\Pi_{1}^{n} p_{i}\left(v_{i}\right)$

D5. $P\left\{f\left(v_{1}, \ldots, v_{n}\right)=u\right\}$
Discrete, $v$ finite or countable, Bernoulli $(v=0,1)$

Poisson

Density for value of a function

Vector density, independent variables

f-value density, independent varlables 
D6. $c_{u}^{n} p^{u}(1-p)^{n-u}$

07. $c_{s-1}^{\nu-1} p^{s}(1-p)^{\nu-s}$

D3. $(1-q) q^{v-1}$

D9. $a^{v-1} /(1+a)^{v}$

D10. $(1+\alpha \beta)^{-1 / \beta}(\alpha / 1+\alpha \beta)^{\mu} x$

$$
(1+\beta) \cdots[1+(\mu-1) \beta] / \mu \text { ! }
$$

D11. $\left(n ! / n_{1} ! \cdots n_{f} 1\right) p_{1} n_{1} \cdots p_{f}^{n_{f}}$

D12. $c_{v}^{M} c_{m-v}^{N} / c_{m}^{M+N}$

D13. $c_{k}^{n} \frac{b \cdots[b+(k-1) s] \cdot c \cdot \cdots[c+(n-k-1) s]}{N \cdot(N+s) \cdot \cdots[N+(n-1) s]}$

D14. $1 / N(N-1) \cdots(N-n+1)$

D15. $1 / C_{n}^{N}$

D16. $\Pi_{i}^{\infty} p_{i}\left(v_{i}\right)$
Binomial, drawing with replacement

Negative binomfal

Geometric

Pascal

Polya $(\beta=1,1 / 2,1 / 3, \ldots)$

Multinomial, macrostate, particles in boxes.

Hypergeometric, drawing without replacement

Polya's urn

Random permutation

Random combination

Random sequences of integers

\section{Discrete Densities}

D1. $P(v) ; v=0,1,2, \ldots$

R. Set $v=\min \left\{k ; \sum_{0}^{k} p(v)>r_{0}\right\}$

D2. $p(v)=e^{-a} a / v l ; v=0,1,2, \ldots, a>0$.

R1. Set $v=\min \left\{n ; \sum_{0}^{n} a^{v} / v !>r_{0} e^{a}\right\}$

R2. Set $v=-1+\min \left\{n ; \frac{n}{\frac{n}{1}} r_{i}<e^{-a}\right\}$

J2. By C2, C7, F7, F3, we have 


$$
\begin{aligned}
& p\left\{r_{1} \cdots r_{n}<e^{-a}\right\}=\left\{\begin{array}{l}
\left.\pi_{1}^{n} r_{1}^{n}<e^{-a}\right\} \quad\left\{\sum_{1}^{n} v_{1}>a, v_{1}>0\right\} \\
\pi_{1}^{n} e^{-v_{1}} d v_{i}
\end{array}\right. \\
& =(1 /(n-1) !) \int_{a}^{\infty} u^{n-1} e^{-u} d u=e^{-a} \sum_{0}^{n-1} a^{i} / 11 \text {. cf. c22. } \\
& \left\{v_{i} f(v)=u\right\}
\end{aligned}
$$

R. Sample $p(v)$ for $v_{\text {; }}$ set $u=f(v)$.

DA. $p(\nu)=p\left(\nu_{1}, \ldots, \nu_{n} L=\pi_{1}^{n} p_{1}\left(\nu_{1}\right)\right.$

R. Sample each $\dot{p}_{1}\left(v_{1}\right)$ for $v_{1}$; set $v=\left(v_{1}, \ldots, v_{n}\right)$

DE. $q(u)=p\left\{f\left(v_{1}, \ldots, v_{n}\right)=u\right\}=\sum_{\{f(v)=u\}} p_{1}\left(v_{1}\right) \cdots p_{n}\left(v_{n}\right)$

R. Sample each $p_{j}\left(v_{j}\right)$ for $v_{1}$; set $u=f\left(v_{1}, \ldots, v_{n}\right)$.

De. $q(u)=c_{u}^{n}(1-p)^{n-u} p^{u} ; u=0,1, \ldots, n, 0<p<1$.

R. Set $u=$ number of $r_{1} \ldots, r_{n}$ such that $r_{i}<p$.

J. For $v_{i} \varepsilon\{0,1\}, p_{j}(0) \equiv 1-p, p_{j}(1) \equiv p, f(v)=v_{1}+\cdots+v_{n}$,

one has $P\left\{f\left(v_{1}, \cdots, v_{n}\right)=u\right\}=\sum p_{1}\left(v_{1}\right) \cdots p_{n}\left(v_{n}\right)$

$\left\{v_{1}+\cdots+v_{n}=u\right\}$

9 


$$
=c_{u}^{n}(1-p)^{n-u} p^{u}
$$

D7. $p(v)=c_{s-1}^{\nu-1} p^{s} q^{\nu-s} ; v=s, s+1, \ldots, 0<p<1, q=1-p, s>1$.

R. Set $v=$ first $n$ for which $s$ of the random numbers $r_{1}, \ldots, r_{n}$ are $<p$.

J. $p(v)=\left\{c_{s-1}^{\nu-1} p^{s-1} q^{(v-1)-(s-1)}\right\}$. $p$ is the probability of exactly $s$ "successes" occurring for the first time at the bath trial.

Note. $\quad 1=p^{s}(1-q)^{-s}=\sum_{0}^{\infty} \frac{(-s)(-s-1) \cdots(-s-\mu+1)}{\mu !} p^{s}(-q)^{\mu}$

$=\sum_{0}^{\infty} \frac{(s+\mu-1) \cdots(s+1) s}{\mu !} p^{s} q^{\mu}=\sum_{0}^{\infty} c_{\mu}^{s+\mu-1} p^{s} q^{\mu}$

$=\sum_{s}^{\infty} c_{v-s}^{\nu-1} p^{s} q^{\nu-s}=\sum_{s}^{\infty} c_{s-1}^{\nu-1} p^{s} q^{\nu-s}$. Hence the term "negative binomial."

De. $g(v)=p q^{v-1} ; v=1,2,3, \ldots, 0<p<1, q=1-p$.

R 1. Set $v=\min \left\{n ; r_{n}<p, n=1,2, \ldots\right\}$.

J 1. Case $s=1$ of 07.

R 2. Set $v=k$, where $k-1<$ en $r_{1} / \ln q<k, k=1,2, \ldots$

J 2. The rule follows from 01 (with $v=1,2, \ldots$ )

For, $\sum_{1}^{k} g(v)=1-q^{k}$. (We have replaced $r_{0}$ by $r_{1}=1-r_{0}$ ).

Dg. $g(v)=a^{\nu-1} /(1+a)^{\nu} ; v=1,2,3, \ldots, a>0$.

10 
R 1. Set $v=\min \left\{n ; r_{n} \leqslant 1 /(1+a), n=1,2, \ldots\right\}$

R. 2. Set $v=k$, where $k-1<\ln r_{1} /\{\ln a-\ln (1+a)\} \leqslant k$.

J. Special case of $D 8$, with $p=1 /(1+a)$.

D10. $h(\mu)=(1+\alpha \beta)^{-1 / \beta}\left(\frac{\alpha}{1+\alpha \beta}\right)^{\mu} \frac{1 \cdot(1+\beta) \cdots(1+(\mu-1) \beta)}{\mu !}$,

$h(0)=(1+\alpha \beta)^{-1 / \beta} ; \mu=0,1,2, \ldots, \alpha>0, \beta=1,1 / 2,1 / 3, \ldots$

R. Define $s=1 / \beta, p=1 /(1+\alpha \beta)$. Set $\mu=$

- $s+\left\{\right.$ first $n$ for which $s$ of the random numbers $r_{1}, \ldots, r_{n}$ are $\left.<p\right\}$.

J. One finds that $h(\mu)=c_{s-1}^{\nu-1} p^{s} q^{\nu-s}$, where $\nu=s+\mu$.

The rule follows from $D 7$.

D11. $p\left(n_{1} \ldots, n_{f}\right)=\left(n ! / n_{1} ! \cdots n_{f} !\right) p_{1}^{n_{1}} \cdots p_{f}^{n_{f}} ;$

$n_{h}>0, n_{1}+\ldots+n_{f}=n, p_{1}+\ldots+p_{f}=1$

R. One follows the steps:

1. Put $0+n_{1}, \ldots, 0 \rightarrow n_{f} ; 1+t$.

2. Set $\left.k=\min \mid k_{i} \sum_{1}^{k} p_{i}>r_{t}\right\}$. Put $1+n_{K}+n_{K}$.

3. If $t<n$, put $t+1 \rightarrow t$, return to (2). Otherwise exit with $\left(n_{1}, \ldots, n_{f}\right)$.

J. If $v_{1}, \ldots, v_{n}$ are independent, each with density given by

$$
\begin{aligned}
v_{i} & =1, \ldots, f \\
p_{i}\left(v_{f}\right) & =p_{1}, \ldots, p_{f}
\end{aligned}
$$


then the vector $\left(v_{1}, \ldots, v_{n}\right)$ has density $p\left(v_{1}, \ldots, v_{n}\right)=$ $p_{v_{1}} \ldots p_{v_{f}}$, where $\sum_{v} p_{v_{1}} \ldots p_{v_{f}}=\left(p_{1}+\ldots+p_{f}\right)^{n}=1$. Hence, the probability, under this density, of the class $C$ of vectors with $n_{1}$ components $1, \ldots, n_{f}$ components $f$, is

$$
\sum_{v \in C} p_{v_{1}} \cdots p_{v_{f}}=\sum_{v \in C} p_{1}^{n_{1}} \cdots p_{f}^{n_{f}}=\left(n ! / n_{1} ! \cdots n_{f} !\right) p_{1}^{n_{1}} \cdots p_{f}^{n_{f}} \text {, }
$$

and the rule follows.

Note. The density $q(u)$ for the sum $u=v_{1}+\cdots+v_{f}$ is not easily expressed (although easily sampled). Only for $f=2$ do we have the connection with the binomial D6.

D12. $p(v)=c_{v}^{M} c_{m-v}^{N} / C_{m}^{M+N} ; \max (0, m-N)<v<\min (m, M), M, N>1,1<m<M+N$.

R. One follows the steps:

1. List the integers $1,2, \ldots, M+N$.

2. Put $0 \rightarrow v, M+N+b, 1 \rightarrow t$.

3. Set $k=\min \left\{k ; k>b r_{t}\right\},(k=1,2, \ldots, b)$.

4. Delete $K^{\text {th }}$ integer $\equiv a_{K}$ of the remaining list. If $a_{K}<M$, put $v+1 \rightarrow v$.

5. If $v=M$, or if $v<M$ and $t=m$, exit with $v$. Otherwise, go to (6).

6. Put $b-1 \rightarrow b, t+1 \rightarrow t$ and return to (3).

3. One notes that $c_{v}^{M} c_{m-v}^{N} / c_{m}^{M+N}=P_{v}^{M} P_{m-v}^{N} \cdot c_{v}^{m} / P_{m}^{M+N}$

where $P_{r}^{n} \equiv n(n-1) \cdots(n-r+1)$; i.e., $p(v)$ is the chance of $m$ drawings without replacement yielding exactly $v$ integers $<M$.

D13. $p(k)=c_{k}^{n} \frac{b(b+s) \ldots(b+(k-1) s) c(c+s) \cdots(c+(n-k-1) s)}{N(N+s) \cdots(N+(n-1) s)}$;

$k=0,1,2, \ldots, n, b, c \in[1,2,3, \ldots\}, \quad N=b+c, s=0,1,2, \ldots$

R. One follows the steps: 
1. Put $0 \rightarrow k, 1 \rightarrow t, b \rightarrow b^{\circ}, N \rightarrow N^{-}$

2. If $r_{t}<b^{-} / N^{-}$, put $k+1 \rightarrow k, b^{-}+s \rightarrow b^{-}$; go to (3). If $r_{t}>b^{-} / N^{-}$, go to (3).

3. Put $N^{-}+s \rightarrow N^{-}$

4. If $t<N$, put $t+1+t$, return to (2). If $t=n$, exit with $k$.

J. $\quad p(k)$ is the probability of drawing $k$ black balls in $n$ trials from an urn initially containing $b$ black and $c$ white balls $(b+c=N)$, subject to the condition:

(c) On the t-th drawing, the ball drawn is replaced, and s more balls of the same color as that drawn are added to the urn. (For $s=0, p(k)$ is the binomial density).

Di4. $P(\pi)=1 / N(N-1) \ldots(N-n+1) ; 1<n<N, \pi=\left\langle C_{1}, \ldots, C_{n}\right)$ permutation of $1, \ldots, \mathrm{s}$, , taken $n$ at a time".

R 1. Follow the steps:

1. List $1,2, \ldots, N$.

2. Put $1+t, N \rightarrow D$

3. Set $k=\min \left\{k ; k>D r_{t}, k=1,2, \ldots, D\right\}$

4. Set $C_{t}=k$-th integer of remaining 1 ist, and delete this integer from list.

5. If $t<n$, put $t+] \rightarrow t, D-1+D$, and return to (3).

Otherwise, exit with random permutation $\pi=\left(c_{1}, \ldots, c_{n}\right)$.

R. 2. (For $n=N$ only!). Follow the steps:

1. Put $1 \rightarrow A_{1}, \ldots, N \rightarrow A_{N} \quad\left(A_{j}\right.$ storage position)

2. Put $1+t, N \rightarrow D$.

3. Set $k=\min \left\{k ; k>D r_{t}, k=1,2, \ldots, D\right\}$

4. Interchange contents of $A_{D}$ and $A_{K^{*}}$ (if $D \neq K$ )

5. If $t<n$, put $t+1+t, D-1 \rightarrow 0$ and return to (3)

Otherwise, exit with random permutation $\pi=\left(c_{1}, \ldots, c_{N}\right)$,

$c_{i}=$ content of $A_{i}$. 
D15. $p(c)=1 / C_{n}^{N} ; 1 \leqslant n \leqslant N, c=\left\{C_{i}, \ldots, C_{n}\right\}$ unordered set of $n$ integers from the set \{\}$, \ldots, N\}$.

R 1. Follow DT4, 1 , and take the set of integers $\left\{C_{7}, \ldots, C_{n}\right\}$ in the permutation $\pi=\left(c_{1}, \ldots, C_{n}\right)$ there obtained.

R 2. Follow the steps:

1. Put $0 \rightarrow m \quad 1 \rightarrow t$

$$
\mathrm{n} \rightarrow \mathrm{C} \quad \mathrm{N} \rightarrow \mathrm{D}
$$

2. If $r_{t}<C / D$, put $\left.m+\right] \rightarrow m$; then set $C_{m}=t$; go to (3). If not go to (6).

3. If $m<n$, go to (4). If $m=n$, go to (9).

4. Put $C-1+C, D-T \rightarrow D$; go to (5).

5. Put $t+1+t$; return to (2).

6. Put $D-1 \rightarrow D$; go to (7).

7. If $C<D$, go to (5). If $C=D$, go to (8).

8. Set $C_{m+1}=t+1, \ldots, C_{n}=N$; go to (9).

9. Exit with random set $c=\left(C_{1}, \ldots, C_{n}\right)$ (arranged in order of magnitude).

J. If $m$ choices have been made through the $t$-th trial, $(t=1,2, \ldots, N-1)$, one accepts $t+1$ as the $m+1-s t$ choice with probability $(n-m) /(N-t)$, where $N-t$ is the number of remaining candidates $t+1, \ldots, N$, and $n-m$ is the number of choices still to be made from these candidates.

D16. $\Pi_{1}^{\infty} p_{i}\left(\nu_{i}\right)$

R. Methods are given in $[7,8]$, based on "Poisson sequences of trials", for producing random sequences of integers of stipulated asymptotic density.

$$
\text { C-INDEX }
$$

\section{Continuous Densities}

cl. $p(v)$

C2. $q(\mu) d \mu=p(\nu) d v$

c3. $\sum_{j}^{J} a_{j}(v)$
General density, continuous on open interval, finite or infinite.

Change of variable.

Sum of positive terms, interpolateú densities. 
C4. $q(u)=\frac{d}{d u} P\{f(v)<u\}$

C5. $\pi_{j}^{n} p_{j}\left(v_{j}\right) d v_{i}$

C6. $q(u)=\frac{d}{d u} P\left\{f\left(v_{1}, \ldots, v_{n}\right)<u\right\}$

C7. $q(u)=F(u) A(u)$

C8. $s(u), p(u), q(u), q_{j}(u)$

c9. $p(v)$

c10. $1 /(b-a)$

c11. $c_{0}+c_{1} \nu$

c12. $c_{0}+c_{1} \nu+c_{2} \nu^{2}$

C13. $u^{m-1}$

C14. $u^{-1}$

C15. $v^{-m-1}$

c16. $(y+1)^{-m-1}$

C17. $e^{-a \nu}$

C18. $e^{-a_{1} u}-e^{-a_{2} u}$

сі9. $\sum_{j}^{n} F_{i} e^{-a_{i} u}$

C20. $\sum_{1}^{n} B_{j} e^{-a_{j} u}$

C21. $B_{1} e^{-a_{1} u}+B_{2} e^{-a_{2} u}$

c22. $u^{n-1} e^{-u}, n=1,2,3, \ldots$

C23. $v^{n-1} /\left(e^{\nu}-1\right), n=2,3, \ldots$

C24. $u^{2 n-1} /\left(e^{u^{2}}-1\right)$

C25. $\nu^{2 n-1} e^{-\nu^{2}}, n=1,2,3, \ldots$

C26. $R e^{-R^{2}}$

C27. $e^{-\nu^{2}},(0, \infty)$

C28. $e^{-v^{2}},(-\infty, \infty)$

c29. $u^{2 n-1} e^{-u^{2}}, n=1 / 2,3 / 2, \ldots$
Density for value of a function.

Vector density, independent variables. f-value density, independent variables. Case $\pi_{1}^{n} p_{i}\left(\nu_{i}\right)=F\left(f\left(v_{1}, \ldots, v_{n}\right)\right)$ of c6. Densities for $v_{1}+v_{2}, v_{1} v_{2}, v_{2} / v_{1}$

A general device. Uniform.

Linear, disk radius

Quadratic, shell radius.

Power $(m>0)$, sphere radius, completely degenerate gas momentum.

Hyperbola

Power $(m>0)$

Pareto ( $m>0$ )

Exponential, Laplace $I$, decay time, collision distance (free path $\lambda=1 / a$ ). Difference of exponentials.

Exponential convolute.

Sum of exponentials $\left(B_{j}>0\right)$.

Hyperexponential, residence times.

I-type, Erlangian

Planck type, Bose-Einstein, photons Version of $\mathbf{C 2 3 .}$

Gauss type, Rayleigh, Maxwell

Gauss type, $n=1$.

Error function

Normal

Gauss type, Maxwell speed. 
C30. $\nu^{2 n-1} /\left(e^{\nu^{2}}-1\right), n=3 / 2,5 / 2, \ldots$. Planck version.

c31. $u^{n-1} /\left(e^{u}-1\right), n=3 / 2,5 / 2, \ldots \quad$ Planck type.

C32. $v^{n-1} e^{-v}, n=1 / 2,3 / 2, \ldots$

I-type, Maxwell energy, fission spectrum.

C33. $y^{A-1} \ell^{n-1}(1 / y)$

Power-log power.

c34. $t^{n p-1} e^{-t^{p}}$

Г-version

C35. $u^{m-1} /(1+u)^{m+n}$

$$
\begin{aligned}
& v^{m-1}(1-v)^{n-1} \\
& \sin ^{2 m-1} \theta \cos ^{2 n-1} \theta \\
& w^{m p-1}\left(1-w^{p}\right)^{n-1}
\end{aligned}
$$

C36. $\exp \left(-\sum_{i}^{N} v_{i}^{2}\right)$

B-types, "arc sin", powers of sin, cos.

C37. $p(\Omega), \Omega=\left(u_{1}, \ldots, q_{n}\right)$

c38. $F\left(\sqrt{\sum_{1}^{N} \xi_{i}^{2}}\right)$

$\mathrm{N}$-normal, Maxwell velocity.

C39. $u^{N / 2-1} e^{-u / 2 b}$

$x^{2}$ density.

C40. $x^{N-1} e^{-x^{2} / 2 b}$

$x$ density, Rayleigh.

C41. $v^{N / 2-1} e^{-N v / 2 b}$

Mean square, $x^{2} / N$.

C42. $x^{N-1} e^{-N x^{2} / 2 b}$

C43. $1 /\left(1+t^{2} / N\right)^{\frac{N+1}{2}}$

Root mean square, $\sqrt{\chi^{2} / N}$.

Student's $t$.

C44. $1 /\left(1+t^{2}\right)$

C45. $F^{M / 2-1} /(1+M F / N)^{\frac{M+N}{2}}$

Cauchy

Snedecor's F

C46. $v^{n-1} e^{-v} /\left(1-\Lambda^{2} e^{-2 v}\right)$

Lemma for R8.

C47. $v^{n-1} e^{-b v},(1, \infty)$

Lemma for R9.

C48. $\nu^{n-1} \Lambda e^{-a \nu} / 1-\Lambda^{2} e^{-2 a \nu}$

Lemma for $R 10$. 
C49. $u^{n-1} \dot{E}_{N}(u)$

C50. $u^{n-1} K_{N}(u)$

c51. $e^{-a|u-b|}$

C52. $x^{b-1} e^{-a x^{b}}$

C53. $u^{-1} \exp \left\{-\left(\ell n^{2} u\right) / 2 b\right\}$

C54. $x^{-(n+1)} e^{-a^{2} / 2 x}$

c55. $s(u)=\left\{\begin{aligned} u ; & 0<u<1 \\ 2-u ; & 1<u<2\end{aligned}\right.$

C56. $\cosh \theta$

C57. $\sinh \theta$

C58. $e^{B x} /(1+B x)^{2}$

C59. $y^{a_{1}-1}-y^{a_{2}-1}$

c60. $\sum F_{i} y^{a_{i}-1}$

C61. $P\left(\alpha^{\circ} / \alpha\right)$
Schlömilch, neutron diffusion.

Bessel

Bilateral exponential, Laplace II.

Weibull

Log normal.

One sided stable, recurrence times.

Triangular.

Hyperbolic

Hyperbol tc

Kahn, approximate normal.

Difference of powers.

Power convolute.

Klein-Nishina total cross section. See $R 7 C, D$ and $R 14$ for psiarized case.

\section{Continuous Densities}

C1. $P(v) ;(a, b)$

R. Define $P(v)=\int_{a}^{v} p(v) d v, p_{1}(v)=\int_{\nu}^{b} p(v) d v$. Set $v=p^{-1}\left(r_{0}\right)$ or $v=p_{1}^{-1}\left(r_{1}\right)$.

C2. $q(\mu) d \mu=p(v) d v ; \mu=f(v)$ monotone

R. If preferable, sample $p(v)$ for $v$; set $\mu=f(v)$. 
Note that $p(v)=q(f(v)) d u / d v$. A similar rule applies to the $n$ variable case, with $d \mu / d v$ replaced by the absolute Jacobian $\left|\operatorname{det}\left[\partial \mu_{i} / \partial \nu_{j}\right]\right|$ of the transformation $\mu=f(v)$.

c3. $\quad p(v)=\sum_{1}^{J} a_{j}(v) ;(a, b), a_{j}(v) \geqslant 0, j$ finite or infinite.

R. Define $A_{j}=\int_{a}^{b} a_{j}(v) d v$. Set $k=\min \left\{k ; \sum_{1}^{k} A_{j} \geqslant r_{0}\right\}$. Sample density $a_{k}(v) / A_{k}$ for $v$.

Note. This provides a quick way of sampling an interpolated density $p(v)=\alpha_{1} p_{1}(v)+\alpha_{2} p_{2}(v), \alpha_{i}>0, \alpha_{1}+\alpha_{2}=1$.

C4. $q(u)=\frac{d}{d u} P\{f(v)<u\}=\frac{d}{d u} \int p(v) d v ;(a, b), p(v)$ density for $v$, $f(v)<u$

$P\{f(v)<a\}=0$ (always assumed:)

R. Sample $p(v)$ for $v$; set $u=f(v)$.

J. Since $\int_{a}^{u} q(u) d u=P\{f(v)<u\}, q(u)$ is the density for the value $u$ of the function $f(v)$.

c5. $p(v) d v=\pi_{i}^{n} p_{i}\left(v_{i}\right) d v_{i}$

R. Sample each $p_{i}\left(v_{i}\right)$ for $v_{i}$. Set $v=\left(v_{1}, \ldots, v_{n}\right)$

C6. $\left.q(u)=\frac{d}{d u} P \mid f\left(v_{1}, \ldots, v_{n}\right)<u\right\}=\frac{d}{d u} \int \Pi_{1}^{n} p_{i}\left(v_{i}\right) d v_{j} ;(a, b)$, $f\left(v_{1}, \ldots, v_{n}\right)<u$

$p_{i}\left(v_{i}\right)$ density for $v_{i}, i=1, \ldots, n$. 
R. Sample each $p_{i}\left(v_{i}\right)$ for $v_{i}$. Set $u=f\left(v_{1}, \ldots, v_{n}\right)$.

C7. $q(u)=F(u) A(u) ;(a, b), \pi_{j}^{n} p_{j}\left(v_{j}\right)=F\left(f\left(v_{j}, \ldots, v_{n}\right)\right), A(u)=d V / d u$, $v(u)=\int_{f(v)<u} \pi_{1}^{n} d v_{i}$.

R. Sample each $p_{i}\left(v_{i}\right)$ for $v_{i}$. Set $u=f\left(v_{1}, \ldots, v_{n}\right)$.

J. If $v_{i}$ has density $p_{j}\left(v_{i}\right)$, and $\pi_{1}^{n} p_{j}\left(v_{i}\right)=F\left(f\left(v_{1}, \ldots, v_{n}\right)\right)$, where $F(u)$ is a function of one variable, and if moreover $A(u)$ is defined as above, then consideration of the one-parameter family of surfaces $f\left(v_{1}, \ldots, v_{n}\right)=u$ leads to the result

$P\{f(v)<u\}=\int_{f(v)<u} \pi_{1}^{n} p_{i}\left(v_{i}\right) \pi_{1}^{n} d v_{i}=\int_{a}^{u} F(u) A(u) d u$.

The rule therefore follows from $\mathrm{c}$.

ce. $s(u)=\int_{0}^{u} p_{1}\left(v_{1}\right) p_{2}\left(u-v_{1}\right) d v_{1} ;(0, \infty), p_{1}, p_{2}$ densities on $(0, \infty)$

$p(u)=\int_{0}^{\infty} p_{1}\left(v_{1}\right) v_{1}^{-1} p_{2}\left(v_{1}^{-1} u\right) d v_{1} ;(0, \infty), p_{1}, p_{2}$ densities on $(0, \infty)$

$q(u)=\int_{0}^{\infty} p_{1}\left(v_{1}\right) \nu_{1} p_{2}\left(v_{1} u\right) d v_{1} ;(0, \infty), p_{1}, p_{2}$ densities on $(0, \infty)$

$q_{1}(u)=\int_{0}^{\infty} p_{1}\left(v_{1}\right) v_{1} p_{2}\left(v_{1} u\right) d v_{1} ;(-\infty, \infty), p_{1}, p_{2}$ densities on $(0, \infty)$ and

$(-\infty, \infty)$, respectively. 
R. In all cases, sample $p_{1}\left(v_{1}\right)$ for $v_{1}, p_{2}\left(v_{2}\right)$ for $v_{2}$. For $s(u)$, set $u=v_{1}+v_{2}$; for $p(u)$ set $u=v_{1} v_{2}$; for $q(u)$ or $q_{1}(u)$, set $u=v_{2} / v_{1}$.

J. In each of the functions $f\left(v_{1}, v_{2}\right)=v_{1}+v_{2}, v_{1} v_{2}, v_{2} / v_{1}$, one verifies that $\frac{d}{d u} \int_{f(v)<u} p_{1}\left(v_{1}\right) d v_{1} p_{2}\left(v_{2}\right) d v_{2}$ has the form of the corresponding density above. Verification for $v_{1}+v_{2}$ is given in $F 2$.

C9. $p(v) ;(a, b)$

R. Define $P(v), P_{1}(v)$ as in $C 1$, and

$$
\begin{array}{ll}
f(r)=r^{-1} P(a+(b-a) r) & \\
g(r)=r^{-1} P_{1}(b-(b-a) r) & 0<r<1 .
\end{array}
$$

(a) If $f(r)$, in particular if $p(v)$, is increasing, set

$$
u=\left\{\begin{array}{l}
r_{1} \text { if } r_{2} \leqslant f\left(r_{1}\right) \\
f^{-1}\left(r_{2}\right) \text { if } r_{2}>f\left(r_{1}\right)
\end{array} \quad v=a+(b-a) u\right.
$$

(b) If $g(r)$ is increasing, in particular if $p(v)$ is decreasing, set

$$
u=\left\{\begin{array}{l}
r_{1} \text { if } r_{2}<g\left(r_{1}\right) \\
g^{-1}\left(r_{2}\right) \text { if } r_{2}>g\left(r_{1}\right)
\end{array} \quad v=b-(b-a) u\right.
$$

J. For example, if $f(r)$ is increasing, and we define the function

$$
F(r, s)=\left\{\begin{array}{l}
r \text { if } s \leqslant f(r) \\
f^{-1}(s) \text { if } s>f(r)
\end{array}\right.
$$

on the unit square, 
then for $p_{1}(r) \equiv 1 \equiv p_{2}(s)$, we have $\int_{0}^{v} q(u) d u \equiv$

$\int p_{1}(r) p_{2}(s) d r d s=v f(v)=P(a+(b-a) v)$

$F(r, s)<v$

$=\int_{a}^{a+(b-a) v} p(v) d v$, and the rule in (a) iollows from $c 6, c 2$.

Note. The method is practical only if $f, g$ are more easily invertible than $P, P_{1}$, which is indeed true for all linear densities, and for quadratic densities on certain intervals (Cf. $\mathrm{Cl1}, 12)$. For details, see [2].

C10. $p(v)=1 /(b-a) ;(a, b)$

R. Set $v=a+(b-a) r_{0}$.

c11. $p(v)=c^{-1}\left(c_{0}+c_{1} v\right) ;(a, b), c_{1} \neq 0, c=(b-a)\left\{c_{0}+\frac{1}{2} c_{1}(a+b)\right\}$.

R. (a) If $c_{1}>0$, set $v=a+\max \left\{(b-a) r_{1},\left(b+a+2 c_{0} c_{1}^{-1}\right) r_{2}-2\left(a+c_{0} c_{1}^{-1}\right)\right\}$.

(b) If $c_{1}<0$, set $v=b-\max \left|(b-a) r_{1},-\left(b+a+2 c_{0}^{-1} c_{1}\right) r_{2}+2\left(b+c_{0} c_{1}^{-1}\right)\right|$.

Note. For $p(v)=2 v /\left(b^{2}-a^{2}\right)$, set $v=a+\max \left\{(b-a) r_{1},(b+a) r_{2}-2 a\right\}$. or $v=\left\{a^{2}+\left(b^{2}-a^{2}\right) r_{0}\right\}^{1 / 2}$ (cl) (radius, uniform circular disk).

c12. $p(v)=c^{-1} p_{1}(v), p_{1}(v)=c_{0}+c_{1} v+c_{2} v^{2} ;(a, b), c_{2} \neq 0$,

$c=(b-a)\left\{c_{0}+\frac{1}{2} c_{1}(b+a)+\frac{1}{3} c_{2}\left(b^{2}+a b+a^{2}\right) \mid\right.$, for certain intervals. 
R. (a) If $c_{2}>0, p_{i}(a)>0$ : or if $c_{2}<0,3 \div-\frac{1}{2} c_{1} c_{2}^{-1}, b<-\frac{1}{2}\left(a+\frac{3}{2} c_{1} c_{2}^{-1}\right)$

$$
\text { set } v= \begin{cases}a+(b-a) r_{1} & \text { if } r_{2}<f\left(r_{1}\right) \\ a+\lambda\left(r_{2}\right) & \text { if } r_{2}>f\left(r_{1}\right)\end{cases}
$$

where $f(r) \equiv(b-a) c^{-1}\left|p_{1}(a)+\frac{1}{2} p_{1}(a)(b-a) r+\frac{1}{3} c_{2}(b-a)^{2} r^{2}\right|$.

$$
\lambda(s) \equiv \frac{1}{2}\left\{-\alpha+\operatorname{sgn} c_{2}{ }^{2} \alpha^{2}+12 c_{2}^{-1}\left(\frac{c}{b-a} s-p_{1}(a)\right)\right\}
$$

and $\alpha \equiv 3\left(a+\frac{1}{2} c_{1} c_{2}^{-1}\right)$

(b) If $c_{2}>0, p_{1}(b)<0$; or if $c_{2}<0, b>-\frac{1}{2} c_{1} c_{2}^{-1}, a>-\frac{1}{2}\left(b+\frac{3}{2} c_{1} c_{2}^{-1}\right)$,

$$
\text { set } v= \begin{cases}b-(b-a) r_{1} & \text { if } r_{2}<g\left(r_{1}\right) \\ b-\mu\left(r_{2}\right) & \text { if } r_{2}>g\left(r_{1}\right)\end{cases}
$$

where $g(r) \equiv(b-a) c^{-1}\left|p_{1}(b)-\frac{1}{2} p_{j}(b)(b-a) r+\frac{1}{3} c_{2}(b-a)^{2} r^{2}\right|$

$$
\mu(s) \equiv \frac{1}{2}\left\{\beta+\operatorname{sgn} c_{2} \checkmark \beta^{2}+12 c_{2}^{-1}\left(\frac{C}{b-a} s-p_{1}(b)\right)\right\}
$$

and $B \equiv 3\left(b+\frac{1}{2} c_{1} c_{2}^{-1}\right)$.

Note. The method, when applicable, is indicated if $\mathrm{Cl}$ involves a difficult cubic. For $p(v)=3 v^{2} /\left(b^{3}-a^{3}\right)$ (radius, uniform spherical shell), $c 1$ sets $v=\left|a^{3}+\left(b^{3}-a^{3}\right) r_{0}\right|^{1 / 3}$, whereas $C 12$ sets

22 
$\nu= \begin{cases}a+(b-a) r_{1} & \text { if } r_{2}<f\left(r_{1}\right) \\ a+\lambda\left(r_{2}\right) & \text { if } r_{2}>f\left(r_{1}\right), \text { where }\end{cases}$

$f(r)=\left\{3 a^{2}+3 a(b-a) r+(b-a)^{2} r^{2}\right\} /\left(b^{2}+b a+a^{2}\right) \equiv \AA+r(B+r \bar{C})$

$\lambda(s)=\frac{1}{2}\left|-3 a+\sqrt{4\left(b^{2}+b a+a^{2}\right) s-3 a^{2}}\right| \equiv D+\sqrt{E s-F} ; A_{i, \ldots, F}$ stored.

Machine times should be compared. For $a=0$, see also $c 13, m=3$.

C13. $q(u)=m b^{-m} u^{m-1} ;(0, b), m=k / l, k, \& \varepsilon\{1,2,3, \ldots\}$

B. Set $u=b\left(\max \left\{r_{1}, \cdots, r_{k}\right\}\right)^{l}$

J. For $\ell=1, m=k$, let $p_{f}\left(v_{f}\right) \equiv 1,1=1, \ldots, k$, and $f(v)=$

$b \max \left\{v_{1}, \ldots, v_{k}\right\}$. Then $\int \pi_{1}^{k} p_{1}\left(v_{1}\right) d v_{1}=b^{-k} u^{k}=\int_{0}^{u} k b^{-k} u^{k-1} d u$. $f(v)<u$

and the rule follows from $c 6$. For $\ell>2$, and $u=b^{1-\ell} v^{\ell}$, note that $q(u) d u=k b^{-k} v^{k-1} d v$, shich reduces the second case to the first.

C13A. $g(u)=c^{-1} u^{m-1} ;(a, b), m$ real $>0, c=\left(b^{m}-a^{m}\right) / m, a>0$.

R. Set $u=\left\{a^{m}+\left(b^{m}-a^{m}\right) r_{0}\right\}^{1 / m}$.

Note. For $m=1 / \ell, a=0$, this gives the rule of $\mathrm{Cl3}$.

C14. $g(u)=c^{-1} u^{-1} ; 0<a<u<b<\infty, c=\ln (b / a)$

R. Set $u=a e^{C r_{0}}$. 
C15. $p(v)=m \beta^{m} v^{-m-1} ; 0<\beta<v<\infty, m=1 / 2,1,3 / 2,2, \ldots$

R. For $m=1,2,3, \ldots$, set $v=\beta / \max \left\{r_{1}, \ldots, r_{m}\right\}$.

For $m=1 / 2,3 / 2, \ldots$, set $v=\beta /\left(\max \left\{r_{1}, \ldots, r_{2 m}\right\}\right)^{2}$.

J. For $v=u^{-1}$, one finds $p(v) d v=m(1 / \beta)^{-m} u^{m-1}(-d u), 0<u<1 / \beta$. and the rule follows from $\mathrm{Cl}$.

C15A. $p(v)=c^{-1} v^{-m-1} ; 0<\beta<u<\alpha<\infty, m$ real $>0, C=\left(\beta^{-m}-\alpha^{-m}\right) / m$.

R. Set $v=1 /\left\{\alpha^{-m}+\left(\beta^{-m}-\alpha^{-m}\right) r_{0}\right\}^{1 / m}$.

C16. $q(y)=m(y+1)^{-(m+1)} ;(0, \infty), m$ real $>0$.

R. Set $y=v-1$, where $v$ is obtained from $C 15$ or C15A with $B=1$.

J. For $y=v-1$, one has $q(y) d y=m v^{-m-1} d v, 1<y<\infty$.

c17. $p(v)=a e^{-a v} ;(0, \infty), a>0$.

R. Set $v=-a^{-1}$ en $r_{0}$

C18. $q(u)=\frac{a_{1} a_{2}}{a_{2}-a_{1}}\left(e^{-a_{1} u}-e^{-a_{2} u}\right) ;(0, \infty), 0<a_{1}<a_{2}$ real.

R. Set $u=-\left(a_{1}^{-1} \ln r_{1}+a_{2}^{-1}\right.$ en $\left.r_{2}\right)$

J. For $p_{1}\left(\nu_{1}\right)=a_{1} e^{-a_{1} v_{1}}, p_{2}\left(\nu_{2}\right)=a_{2} e^{-a_{2} \nu_{2}}$ on $(0, \infty)$, one finds $\int_{0}^{u} p_{1}\left(v_{1}\right) p_{2}\left(u-v_{1}\right) d v_{1}=q(u)$ above. The rule follows from $c 8(s), c 17$. 
C19. $q(u)=\sum_{1}^{n} \frac{\left(a_{1} \cdots a_{n}\right)}{\left(a_{1}-a_{i}\right) \cdots\left(a_{1-1}-a_{i}\right) \cdot\left(a_{i+1}-a_{j}\right) \cdots\left(a_{n}-a_{i}\right)} e^{-a_{i} u} ;(0, \infty)$, $a_{i}>0$, distinct, $n>2$.

R. Set $u=-\sum_{1}^{n} a_{i}^{-1}$ en $r_{i}$.

J. The relation $q(u)=\frac{d}{d u} \int \pi_{1}^{n} a_{j} e^{-a_{q} v_{i}} d v_{i}$ may be proved by induction,

$$
\sum_{1}^{n} v_{1}<u
$$

using $C 18$ as a basis. If $F_{1}^{n}$ denotes the above coefficient of $e^{-a_{1} u}$, the inducton step requires that (CB)

$\int_{0}^{u} \sum_{1}^{n} F_{1}^{n} e^{-a_{f} \nu} d v a_{n+1} e^{-a_{n+1}(u-v)}=\sum_{1}^{n+1} F_{1}^{n+1} e^{-a_{f} u}$. This result follows at once from an identity proved in F10.

C20. $p(u)=\sum_{1}^{n} B_{j} e^{-a_{j} u} ;(0, \infty), B_{j}>0$.

R. Set $k=\min \left\{k_{i} \sum_{1}^{k}\left(B_{j} / a_{j}\right)>r_{0}\right\}$ and $u=-a_{k}^{-1} \ln r_{1}$. (CB, C17).

C21. $p(u)=\left(2 a^{2} / \tau\right) e^{-2 a u / \tau}+2\left((1-a)^{2} / \tau\right) e^{-2(1-a) u / \tau} ;(0, \infty), \tau>0,0<a<1 / 2$.

R. Define $B_{1}=2 a^{2} / \tau, a_{1}=2 a / \tau, B_{2}=2(1-a)^{2} / \tau, a_{2}=2(1-a) / \tau$, whence $B_{1} / a_{1}=a, B_{2} / a_{2}=(1-a)$. Set $K(=1$ or 2$)$ and $u$ as in 620 .

c22. $q(u)=u^{n-1} e^{-u} / \Gamma(n) ;(0, \infty), n=1,2,3, \ldots$

R. Set $u=-\ln \pi_{1}^{n} r_{1}$.

25 
J. For $\dot{p}_{i}\left(v_{i}\right)=e^{-v_{i}},(0, \infty)$, and $f(v)=\sum_{1}^{n} v_{i}$, we have

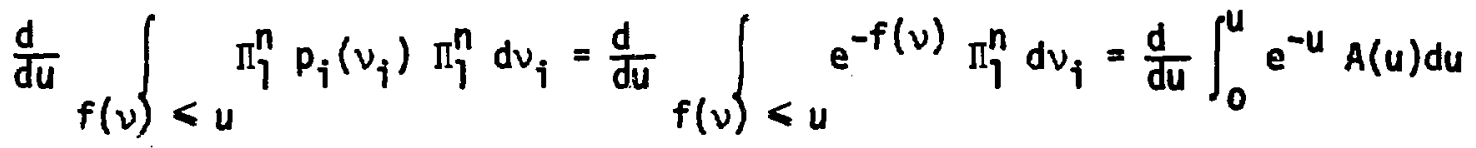

$=e^{-u} A(u)=e^{-u} u^{n-1} /(n-1) !=q(u)$ where $A(u)=d V / d u$

and $V=\int_{f(v)<u} \pi_{1}^{n} d v_{i}=u^{n} / n$ ! as in F7. The rule follows from $\mathrm{C7}, \mathrm{Cl}$.

C23. $p(v)=v^{n-1} /\left(e^{\nu}-1\right) \zeta(n) \Gamma(n) ;(0, \infty), n=2,3,4, \ldots$

R. Set $k=\min \left\{k ; \sum_{1}^{k} j^{-n}>r_{0} \zeta(n)\right\}, v=-k^{-1}$ en $\pi_{1}^{n} r_{\mathfrak{j}}$.

J. Since $\left.p(v) d v=\sum_{1}^{\infty}\left(j^{-n} / \zeta(n)\right) \mid(j u)^{n-1} e^{-j v} d(j v) / \Gamma(n)\right\}$, the rule follows from C3, C22, C2. (Noted for $n=4$ by C. Barnett, E. Canfield of LRL.)

C24. $p(u)=2 u^{2 n-1} /\left(e^{u^{2}}-1\right) \zeta(n) \Gamma(n) ;(0, \infty), n=2,3,4, \ldots$

R. Set $v$ as in $\mathrm{C23}$, and $u=\sqrt{v}$. (C2)

C25. $P(v)=2 v^{2 n-1} e^{-v^{2}} / \Gamma(n) ;(0, \infty), n=1,2,3, \ldots$

R. Set $v=\left\{-\ln \pi_{1}^{n} r_{j}\right\}^{1 / 2} \quad(C 2$, C22)

C26. $P(R)=2 R e^{-R^{2}} ;(0, \infty)$

R. Sat $R=\{-\ln r\}^{1 / 2}$ 
C27. $p\left(v_{1}\right)=2 e^{-v_{1}^{2}} / \sqrt{\pi} ;(0, \infty) \quad$ (See also R12)

R 1. Set $\nu_{1}=R \cos \theta_{1} v_{2}=R \sin \theta$, where $R=\left(-\ln r_{0}\right)^{1 / 2}, \theta=\frac{\pi}{2} r_{1}$. (Two independent samples $v_{1}, v_{2}$ are obtained.)

J. Under the indicated transformation,

$\frac{2}{\sqrt{\pi}} e^{-v_{1}^{2}} d v_{1} \cdot \frac{2}{\sqrt{\pi}} e^{-v_{2}^{2}} d v_{2}=2 R e^{-R^{2}} d R \cdot \frac{2}{\pi} d \theta$. The rule follows from C2, c26.

R 2. obtain $S=r_{1}^{2}+r_{2}^{2}<1$ as in RT. Set

$v_{1}=\left\{\left(-\ln r_{0}\right) / s\right\}^{1 / 2} r_{1}, v_{2}=\left\{\left(-\ln r_{0}\right) / s\right\}^{1 / 2} r_{2}$. (Two samples)

B. Obtain $S=r_{1}^{2}+r_{2}^{2}<1$ as in RI. Set

$v_{1}=\{(-\ln s) / s\}^{1 / 2} r_{1}, v_{2}=\{(-\ln s) / s\}^{1 / 2} r_{2}$. (Two samples)

j3. Under the transformation $\left(\nu_{1}, \nu_{2}\right) \leftrightarrow(\rho, \theta)$ :

$$
v_{1}=R \cos \theta, v_{2}=R \sin \theta, R \equiv\{-2 \ln p\}^{1 / 2},
$$

one finds $\frac{2}{\sqrt{\pi}} e^{-v_{1}^{2}} d v_{1} \frac{2}{\sqrt{\pi}} e^{-v_{2}^{2}} d v_{2}=\frac{4}{\pi} \rho d \rho d \theta$. To sample the latter is to sample the unit disk in quadrant $I$, uniformly in area, and this is Just what $R 1$ does. The method avoids the additional $r_{0}$ of $\$ 2$. (Box, Muller, Marsaglia.)

C28. $P_{0}\left(v_{1}\right)=e^{-v_{1}^{2}} / \sqrt{\pi} ;(-\infty, \infty) \quad$ (See also R/2)

i1. Set $v_{1}=R \cos \theta, v_{2}=R \sin \theta$, where $R=\left(-\ell n r_{0}\right)^{1 / 2}, \theta=2 \pi r_{1}$.

R 2. Obtain $S<1, x, y$ as in $23, B$. Set 
$v_{1}=\left\{\left(-\ln r_{0}\right) / s\right\}^{1 / 2} x, \quad v_{2}=\left\{\left(-\ln r_{0}\right) / s\right\}^{1 / 2} y$.

R. Obtain $S<1, x, y$ as in R3, 3 . Set

$$
v_{1}=\{(-\ln s) / s\}^{1 / 2} x, \quad v_{2}=\{(-\ln s) / s\}^{1 / 2} y .
$$

J. Cf. C27.

Note. Two independent samples $v_{1}=v_{2}$ are obtained in each rule.

C29. $p(u)=2 u^{2 n-1} e^{-u^{2}} / \Gamma(n) ; \quad(0, \infty), n=1 / 2,3 / 2,5 / 2, \ldots$

B. Define $h=n-1 / 2(h=0,1,2, \ldots)$. Sample $2 e^{-\tau^{2}} / \sqrt{\pi}$ for $(\tau)^{2}$ by any of the rules in C27. (Two samples. Save one. Avoid squaring square roots!). Set

$$
u=\left|-\ln \pi_{j}^{h} r_{i}+(\tau)^{2}\right|^{1 / 2}
$$

J. For $p_{i}\left(v_{i}\right)=2 e^{-v_{i}^{2}} / \sqrt{\pi}$ on $(0, \infty), i=1, \ldots, 2 n$, and

$f(v)=\left|\sum_{1}^{2 n} v_{i}^{2}\right|^{1 / 2}$, one has $\pi_{1}^{2 n} p_{i}\left(v_{i}\right)=2^{2 n} e^{-f^{2}(v) / \pi^{n}}$

$A=\frac{d}{d u} \int_{f(u)<u} \pi_{1}^{2 n} d v_{i}=\pi^{n} u^{2 n-1} / 2^{2 n-1} \Gamma(n), \quad(F 8)$, and hence the rule follows

fran $\mathrm{C7}, \mathrm{C27}$.

C30. $p(v)=2 v^{2 n-1} /\left(e^{v^{2}}-1\right) \zeta(n) \Gamma(n) ;(0, \infty), n=3 / 2,5 / 2, \ldots$

B. Set $k=\min \left\{k ; \sum_{7}^{k} j^{-n}>r_{0} \zeta(n)\right\}, h=n-l / 2$.

Sample $2 e^{-\tau^{2}} / \sqrt{\pi}$ for $(\tau)^{2}$ by C27 (two samples, save one. Avoid squaring square roots.) Set 


$$
v=\left\{K^{-1}\left\{-\ln \pi_{1}^{h} r_{f}+(\tau)^{2}\right\}\right\}^{1 / 2} \text {. (C3, C29. Cf. C23) }
$$

C31. $P(u)=u^{n-1} /\left(e^{u}-1\right) \zeta(n) \Gamma(n) ;(0, \infty), n=3 / 2,5 / 2, \ldots$

R. Obtain $(v)^{2}$ as in C30. (Avoid squaring the square rootl) Set $u=(v)^{2}$.

c32. $p(v)=v^{n-1} e^{-v} / \Gamma(n) ; \quad(0, \infty), n=1 / 2,3 / 2,5 / 2, \ldots$

R. Obtain $(u)^{2}$ as in C29. (Avold squaring the square root.) Set $v=(u)^{2}$. (c2, (29).

C33. $g(y)=A^{n} y^{A-1} \ell n^{n-1}(1 / y) / \Gamma(n) ; \quad(0,1), A>0, n=1 / 2,1,3 / 2,2, \ldots$

R. Set $y=e^{-x / A}$, where $x$ is the $u$ of C22 or the $v$ of C32.

J. $g(y) d y=x^{n-1} e^{-x}(-d x) / \Gamma(n)$.

C34. $g(t)=p t^{n p-1} e^{-t^{p}} / \Gamma(n) ;(0, \infty), p$ real $>0, n=1 / 2,1,3 / 2,2, \ldots$

R. Sample $x^{n-1} e^{-x} / \Gamma(n)$ for $x$ on $(0, \infty)$ by C22 or $C 32$.

Set $t=x^{1 / p} . \quad$ (C2) For $p=2$, ef. C25, c29.

C35. $b(u)=u^{m-1} /(1+u)^{m+n} \cdot B(m, n) ; \quad(0, \infty)$

$p(v)=v^{m-1}(1-v)^{n-1} / B(m, n) ; \quad(0,1)$

$q(\theta)=2 \sin ^{2 m-1} \theta \cos ^{2 n-1} e / B(m, n) ; \quad(0, \pi / 2)$

$c(w)=p w^{m p-1}\left(1-w^{p}\right)^{n-1} / B(m, n) ; \quad(0 ; 1), p$ real $>0$

$m, n \in\{1 / 2,1,3 / 2,2, \ldots\}$ in all. 
R. Sample $p_{1}\left(v_{1}\right)=v_{1}^{n-1} e^{-v_{1}} / \Gamma(n), p_{2}\left(v_{2}\right)=v_{2}^{m-1} e^{-v_{2}} / \Gamma(m)$

for $v_{1}, v_{2}$ on $(0, \infty)$ by C22 and/or C32. Set $u=v_{2} / v_{1}, v=u /(1+u)$,

$\theta=\operatorname{arc} \sin \sqrt{\nu_{0}} w=v^{1 / p}$. (The densities are equivalent under the last 3 substitutions.)

J. The rule follows from $\mathrm{Cs}(q)$, since

$\int_{0}^{\infty} p_{1}\left(v_{1}\right) v_{1} p_{2}\left(u v_{1}\right) d v_{1}=b(u)$ above. Note. The same rule results from the equivalence $\frac{2}{\Gamma(n)} \xi^{2 n-1} e^{-\xi^{2}} d \xi \cdot \frac{2}{\Gamma(m)} n^{2 m-1} e^{-n^{2}} d n=$ $\frac{2}{\Gamma(m+n)} \rho^{2(m+n)-1} e^{-\rho^{2}} d \rho \cdot \frac{2}{B(m, n)} \sin ^{2 m-1} \theta \cos ^{2 n-1} \theta d \theta$. Note. For $n=1, m=1 / 2,1,3 / 2,2, \ldots$, see $c 13$.

For $n=1 / 2$, observe that $q(\theta)=2 \Gamma\left(m+\frac{1}{2}\right) \sin ^{2 m-1} \theta / \sqrt{\pi} \Gamma(m)$, $m=1 / 2,1,3 / 2,2, \ldots$ For $n=1 / 2=m$, set $\theta=\frac{\pi}{2} r_{0}, v=\sin ^{2} \theta$, etc.

C36. $p\left(v_{1}, \ldots, v_{N}\right)=\pi^{-N / 2} \exp -\sum_{1}^{N} v_{i}^{2} ;-\infty<v_{i}<\infty, N=1,2, \ldots$

R. For $N=2 h$, obtain $\left(v_{1}, v_{2}\right), \ldots,\left(v_{2 h-1}, v_{2 h}\right)$ from C28.

For $N=2 h+1$, obtain also $\left(v_{2 h+7}, v_{2 h+2}\right)$ from c28 (save $\left.v_{2 h+2}\right)$. 
Note. The density of the value $u$ of the function $f(v)=\left(\sum_{1}^{N} v_{1}^{2}\right)^{1 / 2}$ is $2 u^{N-1} e^{-u^{2}} / \Gamma(N / 2) \quad$ (Cf. C40).

c37. $p(\Omega)=\Gamma(N / 2) / 2 \pi^{N / 2}$

R. Set $v_{1}, \ldots, v_{N}$ as in $C 36$, and $\Omega=\left(\omega_{1}, \ldots .4 w_{N}\right)$.

where $\omega_{i}=v_{j} / p, p=\left(\sum_{1}^{N} v_{i}^{2}\right)^{1 / 2}$.

Note 1. In (28 (the source of the $v_{1}$ ), observe that $v_{1}^{2}+v_{2}^{2}=-\ln r_{0}$ or $-\ln S$; this saves time in computing $\rho$.

Note 2. The rule determines a uniformly distributed direction $\Omega$ in N-space; equivalently, a point on the unit sphere $|\Omega|=1$ (F8). See also R3, 4, 5 .

C38. $p\left(\xi_{1}, \ldots, \xi_{N}\right)=F(u)_{i} u=\left(\sum_{1}^{N} \xi_{1}^{2}\right)^{1 / 2},-\infty<\xi_{1}<\infty$.

R. Sample $q(u)=2 \pi^{N / 2} u^{N-1} F(u) / \Gamma(N / 2)$ for $u$ on $(0, \infty)$.

Sample unit sphere $|.2|=1$ for $\Omega$ by $C 37$. Set $\xi_{j}=u \omega_{j}$.

J. $\left.\quad \int_{i}^{N} \xi_{i}^{2}\right)^{1 / 2}<u$

Note. For $F(u)$ normal, use 036 .

c39. $q(u)=u^{\frac{N}{2}-1} e^{-u / 2 b} /(2 b)^{N / 2} r(N / 2) ;(0, \infty), b>0, N=1,2,3, \ldots$

B. Sample $w^{N / 2-1} e^{-w / \Gamma(N / 2)}$ for $w$ on $(0, \infty)$ by C22 or C32. 
Set $u=2 b w . \quad$ (c2).

Note. $q(u)$ is the density of the value of $u=x^{2}=\sum_{1}^{N} v_{j}^{2}$, defined in statistics as $\frac{d}{d u} \int_{\sum_{1}^{N} v_{i}^{2}<u} \pi_{1}^{N} \frac{1}{\sqrt{2 \pi b}} e^{-v_{i}^{2} / 2 b} d v_{i},-\infty<v_{i}<\infty$, which may be evaluated using $\mathrm{F8}, \mathrm{C7}$.

c40. $p(x)=2 x^{N-1} e^{-x^{2} / 2 b} /(2 b)^{N / 2} \Gamma(N / 2) ;(0, \infty), b>0, N=1,2,3, \ldots$

R. Sample $w^{N / 2-1} e^{-w / r(N / 2)}$ by c22 or C32. Set $x=\sqrt{2 b w}$.

Note. $p(x)$ is the density for $x=\sqrt{\sum_{1}^{N} v_{i}^{2}}$.

c41. $g(v)=N^{N / 2} v^{N / 2-1} e^{-N v / 2 b} /(2 b)^{N / 2} \Gamma(N / 2) ;(0, \infty), b>0, N=1,2,3, \ldots$

19. Sample $w^{N / 2-1} e^{-W / \Gamma(N / 2)}$ by $c 22$ or C32. Set $v=2 b w / N$

Note. $q(v)$ is the density of the mean square $\sum_{1}^{N} v_{i}^{2} / N=x^{2} / N$.

C42. $p(x)=2 N^{N / 2} x^{N-1} e^{-N x^{2} / 2 b} /(2 b)^{N / 2} \Gamma(N / 2) ;(0, \infty), b>0, N=1,2,3, \ldots$

B. Sample $w^{N / 2-1} e^{-W / \Gamma(N / 2)}$ by C22 or C32. Set $x=\sqrt{2 b w / N}$ (C2)

Note. $p(x)$ is the density" of the "root mean square" $\sqrt{\sum_{1}^{N} v_{i}^{2} / N}$

$=\sqrt{x^{2} / N}=x / \sqrt{1}$.

C43. $q_{1}(t)=\Gamma\left(\frac{N+1}{2}\right) / \sqrt{1 \pi} \quad r(N / 2)\left(1+\frac{t^{2}}{N}\right)^{\frac{N+1}{2}} ;(-\infty, \infty), N=1,2,3, \ldots$ 
R. Sample $w^{N / 2-1} e^{-W / \Gamma(N / 2)}$ for $w$ on $(0, \infty)$ by $C 22$ or $C 32$;

set $x=(w / N)^{1 / 2}$. Sample $e^{-y^{2}} / \sqrt{\pi}$ for $y$ on $(-\infty, \infty)$ by C28. Set $t=y / x$.

J. $q_{1}(t)$ is the density for the function $\left.y / \mid N^{-1} \sum_{1}^{N} v_{i}^{2}\right\}^{1 / 2}$ where the $v_{i}, y$ al1 have density $e^{-\xi^{2} / 2 b} / \sqrt{2 \pi b}$ on $(-\infty, \infty)$. For, using the density $P_{0}(y)=e^{-y^{2} / 2 b} / \sqrt{2 \pi b}$ for $y$ and the $p(x)$ of $C 42$ for $x=\sqrt{N^{-1} \sum_{1}^{N} v_{1}^{2}}$, one finds that $\int_{0}^{\infty} p(x) x p_{0}(t x) d y=q_{1}(t)$ above. The result being independent of $b$, the rule follows from $C 8, C 28, c 42$ (with $2 b=1$ ).

C44. $q(t)=1 / \pi\left(1+t^{2}\right):(-\infty, \infty)$

R. Set $t=\tan \frac{\pi}{2}\left(2 r_{0}-1\right)$, or $t=y / x$ as in $R 2$.

Note. Compare with $\mathrm{C} 43(N=1)$.

C45. $q(F)=(M / N)^{M / 2} F^{\frac{M-2}{2}} /\left(1+\frac{M}{N} F\right)^{\frac{M+N}{2}} B(M / 2, N / 2) ;(0, \infty), M, N \varepsilon\{1,2,3, \ldots\}$

R. Define $m=M / 2, n=N / 2$. Sample $v_{1}^{n-1} e^{-v_{1}} / r(n), v_{2}^{m-1} e^{-v_{2}} / \Gamma(m)$ for $v_{1}, v_{2}$ on $(0, \infty)$ by C22 and/or C32. Set $F=N v_{2} / M v_{1}$.

J. For $F=N u / M$, one finds $q(F) d F=b(u) d u$ as in $C 35$. Note. $q(F)$ is the density for the function $M^{-1} \sum_{1}^{M} \mu_{i}^{2} / N^{-1} \sum_{1}^{N} v_{j}^{2}$, where all $\mu_{j}, \nu_{j}$ have density $e^{-\xi^{2} / 2 b} / \sqrt{2 \pi b}$ on $(-\infty, \infty)$. Using the densities $q_{M}(u), q_{N}(v)$ of $C 41$ for numerator and denominator, it is easy to verify that $\int_{0}^{\infty} q_{N}(v) v q_{M}(F v) d v=q(F)$ above, independent of b. Cf. $c 8$. 
C46. $q(v)=c^{-1} v^{n-1} \Lambda e^{-v} /\left(1-\Lambda^{2} e^{-2 v}\right) ;(0, \infty), 0<\Lambda<1, n=3 / 2,2,5 / 2,3, \ldots$

$c=\zeta_{u}(\Lambda, n) \Gamma(n), \zeta_{u}(\Lambda, n) \equiv \sum_{1}^{\infty} \Lambda^{2 j-1} /(2 j-1)^{n}$

R. Set $k=\min \left\{k ; \sum_{1}^{k} \Lambda^{2 j-1} /(2 j-1)^{n}>r_{0} \zeta_{u}(\Lambda, n)\right\}$. Sample $x^{n-1} e^{-x} / \Gamma(n)$ for $x$ on $(0, \infty)$ by C22 or C32. Set $y=x /(2 K-1)$.

J. One can write $q(v)=\sum_{1}^{\infty}\left\{\Lambda^{2 j-1} /(2 j-1)^{n} \zeta_{u}(\Lambda, n)\right\}$. $(2 j-1)^{n} v^{n-1} e^{-(2 j-1) v} d v / \Gamma(n)$ and apply $c 3, c 2$.

C47. $q(v)=D_{b}^{-1} v^{n-1} e^{-b v} ;(1, \infty), b>0, n=1,2,3, \ldots$

$D_{b}=(n-1) ! b^{-n} e^{-b} s_{b}, s_{b}=\sum_{0}^{n-1} b^{i} / i !$

R. Set $L=\min \left\{l ; \sum_{0}^{l} b^{i} / i !>r_{0} S_{b}\right\} \quad(0<<<-1)$, and $v=1-b^{-1}$ en $\pi_{1}^{n-L} r_{i}$

J. Under the transfornation $v=1+b^{-1} u$, one finds $q(v) d v=p(u) d u$ $\equiv 0_{b}^{-1} b^{-n} e^{-b}(u+b)^{n-1} e^{-u_{d u}}=\sum_{0}^{n-1}\left|s_{b}^{-1} b^{i} / i !\right| u^{n-i-1} e^{-u} d u /(n-i-1) ! \quad, 0<u<\infty$, and the rule follows from $\mathrm{C3}, \mathrm{C22}$.

Note. The partial sums of $S_{b}$ should be stored.

C48. $\bar{q}(v)=D^{-1} v^{n-1} \Lambda e^{-a v} /\left(1-\Lambda^{2} e^{-2 a v}\right) ;(1, \infty), 0<\Lambda<1, n=2,3, \ldots$,

$D=\sum_{1}^{\infty} \Lambda^{2 j-1} D_{(2 j-1) a}, D_{b} \equiv(n-1) ! b^{-n} e^{-b} s_{b}, s_{b}=\sum_{0}^{n-1} b^{1} / 1 !$ 
1R. Compute and store partial sums $S_{k}$ of $S=\sum_{1}^{\infty} \Lambda^{2 j-1}(2 j-1)^{-n} e^{-2 j a} S_{(2 j-1) a}$, where $S_{b}$ is defined above.

Set $K=\min \left\{k ; s_{k}>S r_{0}\right\}$. Use $C 47$, with $b \equiv(2 K-1) a$, to obtain $v$ on $(1, \infty)$.

C49. $q(u)=C^{-1} u^{n-1} E_{N}(u) ;(0, \infty), \quad \infty 0, n+N>1, n=1 / 2,1,3 / 2,2, \ldots$, $E_{N}(u)=\int_{1}^{\infty} \nu^{-N} e^{-u \nu} d v, C=\Gamma(n) /(n+N-1) . \quad(F 18,19)$.

R. Sample $p_{1}(x)=(n+N-1) x^{n+N-2}$ for $x$ on $(0,1)$ by $C 13$, and $p_{2}(y)=y^{n-1} e^{-y} / \Gamma(n)$ for $y$ on $(0, \infty)$ by C22 or C32. Set $u=x y$.

J. $\quad \frac{d}{d u} \int_{x y<u} p_{1}(x) d x p_{2}(y) d y=\frac{d}{d u} \int_{0}^{1} p_{1}(x) d x \int_{0}^{u / x} p_{2}(y) d y$

$=\int_{0}^{1} p_{1}(x) d x x^{-1} p_{2}(u / x)=c^{-1} u^{n-1} \int_{0}^{1} x^{N-2} e^{-u / x} d x=q(u)$

The rule follows from $\mathrm{C} 6$. (Cf. C8p)

Note. For $N=0, n=3 / 2,2,5 / 2, \ldots$, sample $q(u)=u^{n-2} e^{-u} / \Gamma(n-1)$ for $u$ on $(0, \infty)$ by $C 22$ or $C 32$.

C50. $q(u)=c^{-1} u^{n-1} k_{N}(u) ;(0, \infty), N=0,1 / 2,1,3 / 2,2, \ldots$,

$n-N=1,2,3, \ldots, k_{N}(u)=\int_{0}^{\infty} \cosh N \theta e^{-u \cosh \theta} d \theta$,

$C=2^{n-2} \Gamma\left(\frac{n-N}{2}\right) \Gamma\left(\frac{n+N}{2}\right)$.

R 1. Define $H=(n-N) / 2, J=(n+N) / 2$. Sample $p_{1}(x)=x^{H-1} e^{-x} / \Gamma(H)$ 
and $p_{2}(x)=x^{J-1} e^{-x} / \Gamma(J)$ for $x, y$ on $(0, \infty)$ by C22 and/or C32.

Set $u=2(x y)^{1 / 2}$.

J1. Transforming by $u=2 v^{1 / 2}$, we have $q(u) d u=c^{-1} u^{n-1} K_{N}(u) d u$ $=c^{-1} 2^{n-1} v^{\frac{n}{2}-1} k_{N}\left(2 v^{1 / 2}\right)=\int_{0}^{\infty} p_{1}(x) x^{-1} p_{2}\left(x^{-1} v\right) d x \quad$ (cf. $\left.F 13(d)\right)$, and the rule follows from C2, C8p.

Note. The Legendre relation $2^{n-1} \Gamma\left(\frac{n}{2}\right) \Gamma\left(\frac{n+1}{2}\right)=\Gamma\left(\frac{1}{2}\right) \Gamma(n)$ may be regarded as a consequence of the above reduction!

R. Define $H=(n-N) / 2, K=N+\frac{1}{2}$. Sample $p(\xi)=\xi^{H-1}(1-\xi)^{K-1} / B(H, K)$ for $\xi$ on 10,1$)$ by $C 35$, and $p_{2}(y)=y^{n+N-1} e^{-y} / \Gamma(n+N)$ for $y$ on $(0, \infty)$ by $C 22$. Set $u=y \xi^{1 / 2}$.

32. Define $p_{1}(x)=\frac{2}{B(H, K)} x^{n-N-1}\left(1-x^{2}\right)^{N-1 / 2}$. Then by $F 13$, we may write $q(u)=\int_{0}^{1} p_{1}(x) x^{-1} p_{2}\left(x^{-1} u\right) d x=\frac{d}{d u} \int_{x y<u} p_{1}(x) d x p_{2}(y) d y$. Hence, we can sample $p_{1}(x)$ for $x$ on $(0,1)$ and $p_{2}(y)$ on $(0, \infty)$, and set $u=x y$, by c8p. But for $x=\xi^{1 / 2}$, we have $p_{1}(x) d x=p(\xi) d \xi$, as defined above; so we may sample $p(\xi)$ for $\xi$ on $(0,1)$ and set $x=\xi^{1 / 2}$. The rule follows. (Noted by Kalos [11] for $n=N+2$.

C57. $q(u)=(a / 2) \exp -a|u-b| ;(-\infty, \infty), a>0,-\infty<b<\infty$.

R. Set $u=b \pm a^{-1}$ en $r_{0}$, the signs being equally likely. (C2, C17) 
C52. $f(x)=a b x^{b-1} e^{-a x^{b}} ;(0, \infty), a, b>0$.

R. Set $x=\exp \left\{b^{-1} \ln \left(-a^{-1} \ln r_{0}\right)\right\} \quad$. (C2, C17)

C53. $q(u)=u^{-1} \exp \left\{-\left(\ln ^{2} u\right) / 2 b\right\} /(2 n b)^{1 / 2} ;(0, \infty), b>0$.

R. Sample $e^{-v^{2}} / \sqrt{\pi}$ for $v$ on $(-\infty, \infty)$ by C28. Set $u=e^{v \sqrt{2 D}}$.

J. For $u=e^{v \sqrt{2 b}}$, one has $q(u) d u=e^{-v^{2}} d v / \sqrt{\pi}$.

Note. Since $q(u)=\frac{d}{d u} \int_{e^{x}<u} e^{-x^{2} / 2 b} d x /(2 \pi b)^{1 / 2}, q(u)=\frac{d}{d u} P\left\{e^{x}<u\right\}$

under the density $e^{-x^{2} / 2 b} /(2 \pi b)^{1 / 2}$.

C54. $f(x)=a^{2 n} x_{x}^{-(n+1)} e^{-a^{2} / 2 x} / 2^{n} \Gamma(n) ;(0, \infty), a>0, n=1 / 2,1,3 / 2,2, \ldots$

R. Sample $y^{n-1} e^{-y} / \Gamma(n)$ for $y$ on $(0, \infty)$ by C22 or c32. Set $x=a^{2} / 2 y$.

C55. $s(u)=\left\{\begin{aligned} & u ; 0<u<1 \\ & 2-u ; 1<u<2 \\ & \hline\end{aligned}\right.$

R. Set $u=r_{1}+r_{2}$.

J. For $p_{1}\left(v_{1}\right) \equiv 1 \equiv p_{2}\left(v_{2}\right), v_{i}$ on $(0,1)$, it is geometrically obvious that

$P\left\{v_{1}+v_{2}<u\right\}=\left\{\begin{array}{l}u^{2} / 2 ; 0<u<1 \\ 1-(2-u)^{2} / 2 ; \quad 1<u<2\end{array}\right.$

Hence, $\frac{d}{d u} p\left\{v_{1}+v_{2}<u\right\}=s(u)$, and the rule follows from $c 6$.

C56. $p(\theta)=s^{-1} \cosh \theta ;(0, t), s=\sinh t$. 
Br. Define $A_{1}=\left(e^{t}-1\right) / 2 S, A_{2}=\left(1-e^{-t}\right) / 2 S$. Set $k=\min \left(k ; \sum_{1}^{k} A_{j}>r_{0}\right\}$ $(K=1$ or 2$)$. For $K=1$, set $\theta=\ln \left\{1+r_{1}\left(e^{t}-1\right)\right\}$. For $K=2$, set $\theta=-\ln \left\{1-r\left(1-e^{-t}\right)\right\} \quad(C 3, C 1)$.

R2. Set $\theta=\ln \left\{s r_{0}+\sqrt{\left(S r_{0}\right)^{2}+1}\right\}$

C57. $p(\theta)=c^{-1} \sinh \theta ;(0, t), c=\cosh t-1$

R. Set $\theta=\ln \left\{\left(\left(r_{0}+1\right)+\sqrt{\left(\left(r_{0}+1\right)^{2}-1\right\}}\right.\right.$

J. The rule follows from $\mathrm{Cl}$. Note that the (t) sign is required, since $x \equiv e^{\theta}>1$ and $a=\left(x+x^{-1}\right) / 2<(x+x) / 2=x$.

C58. $f(x)=B e^{B x} /\left(1+e^{B x}\right)^{2} ;(-\infty, \infty), B>0$.

R. Set $x=B^{-1} \ln \left(r_{0}^{-1}-1\right)$

C59. $p(y)=\frac{a_{1} a_{2}}{a_{2} a_{1}}\left(y^{a_{1}-1}-y^{a_{2}-1}\right) ;(0,1), 0<a_{1}<a_{2}$

R. Set $y=\exp \left\{a_{1}^{-1} \ln r_{1}+a_{2}^{-1} \ln r_{2}\right\}$

J. For $y=e^{-u}$, one finds $p(y) d y=q(u) d u$ as in $c 18$.

c60. $p(y)=\sum_{1}^{n} \frac{\left(a_{1} \ldots a_{n}\right) y^{a_{j}-1}}{\left(a_{1}-a_{j}\right) \ldots\left(a_{j-1}-a_{j}\right) \cdot\left(a_{i+1}-a_{f}\right) \ldots\left(a_{n}-a_{j}\right)}$;

$(0,1), a_{i}$ distinct, $n \geq 2$. 
R. Set $y=\exp \sum_{1}^{n} a_{i}^{-1}$ en $r_{i}$

J. For $y=e^{-u}$, one has $p(y) d y=q(u) d u$ as in $c 19$.

C61. $P\left(\alpha^{\prime} / \alpha\right)$

R. A direct method is given in $[5,6]$ for sampling the $\mathrm{Klein-Nishina}$ differential cross section, based on $\mathrm{Cl}$, and an accurate fit for the inverse of the associated probability distribution.

\section{R-INDEX}

\section{Rejection Techniques}

R1. $\cos \theta, \sin \theta ; p(\theta)=2 / \pi,(0, \pi / 2)$ Uniform direction, quadrant $I$.

R2. $\cos \theta, \sin \theta, \tan \theta ; p(\theta)$ $=1 / \pi,(-\pi / 2, \pi / 2)$

R3. $\cos \theta, \sin \theta ; p(\theta)=1 / 2 \pi,(0,2 \pi)$ Uniform direction in $p l a n e$, point on unit circle.

R4. $\Omega=\left(w_{1}, w_{2}, w_{3}\right)$

Uniform direction in 3-space, point on unit sphere.

R5. $\Omega=\left(\omega_{1}, \ldots, \omega_{N}\right)$

Uniform direction in $\mathrm{N}$-space, point on unit sphere $|\Omega|=1$.

R6. $P_{1}(x)\left\{P_{2}(h(x))-P_{2}(g(x))\right\}$

Density $*$ distribution

R7. $p_{1}(x) h(x)$

Density $x$ bounded function, various versions R7A-D.

R8. $v^{n-1} /\left(\Lambda^{-1} e^{v}+1\right) ; n=3 / 2,1,5 / 2,2, \ldots$ (NR) non-degenerate electron gas energy.

R9. $x^{n-1} e^{-b \sqrt{x^{2}+1}} ; n=2,3, \ldots$

(R) extreme non-degenerate electron gas momentum, Maxwe 11-Juttner.

R10. $x^{n-1} /\left(\Lambda^{-1} e^{a \sqrt{x^{2}+1}}+1\right) ; n=2,3, \ldots$

(R) non-degenerate electron gas momentum. 
R11. $e^{-\mathrm{aE}} \sinh \sqrt{\mathrm{bE}}$

R12. $e^{-x^{2} / 2}$

R13. $P\left(\alpha^{\prime} / \alpha, \theta\right)$
Fission eriergy spectrum, Cranberg density.

Normal.

Polarized Klein-Nishina.

\section{Rejection Techniques}

Note. In all cases, the process is iterated until the stated condition is satisisied.

Ei. $\cos \theta, \sin \theta$ for $p(\theta)=2 / \pi ;(0, \pi / 2)$

B. If $S \equiv r_{1}^{2}+r_{2}^{2}<1$, set $\cos \theta=r_{1} / S^{1 / 2}, \sin \theta=r_{2} / S^{1 / 2}$.

R2. $\cos \theta, \sin \theta, \tan \theta$ for $p(\theta)=1 / \pi ;(-\pi / 2, \pi / 2)$

B. If $S \equiv x^{2}+y^{2}<1$, where $x=r_{1}, y=2 r_{2}-1$, set

$\cos \theta=x / 5^{1 / 2}, \sin \theta=y / s^{1 / 2}$, and $\tan \theta=y / x \quad(x \neq 0)$.

R3. $\cos \phi, \sin \phi$ for $p(\phi)=1 / 2 \pi ;(0,2 \pi)$

R1. If $S \equiv x^{2}+y^{2}<1$, where $x=2 r_{1}-1, y=r_{2}$, set

$\cos \phi=\left(x^{2}-y^{2}\right) / S, \sin \phi=2 x y / S \quad$ (von Neumann)

R2. Use R1 to obtain $\cos \phi$, sin $\phi$; change sign of each independently with probability $1 / 2$.

B3. If $S \equiv x^{2}+y^{2}<1$, where $x=2 r_{1}-1, y=2 r_{2}-1$,

set $\cos \phi=x / S^{1 / 2}, \sin \phi=y / s^{1 / 2}$. 
R4. Uniform direction $\Omega=\left(\omega_{1}, \omega_{2}, \omega_{3}\right)$ in 3-space

Ri. Obtain $\cos \phi, \sin \phi$ from R3. Set $\cos \theta=2 r_{3^{*}}$, $\sin \theta=+\left(1-\cos ^{2} \theta\right)^{1 / 2}, \Omega=(\sin \theta \cos \phi, \sin \theta \sin \phi, \cos \theta)$.

J1. For spherical coordinates, $p(\theta, \phi) d \theta d \phi=$

$\frac{1}{2} \sin \theta d \theta \cdot \frac{1}{2 \pi} d \phi ; p_{1}(\theta) d \theta=\frac{1}{2} \sin \theta d \theta=-\frac{1}{2} d(\cos \theta)=-\frac{1}{2} d \mu ;-1<\mu<1$.

R2. Obtain S, $\cos \phi, \sin \phi$ from R3. Set $\cos \theta$

$=2 S-1, \sin \theta=+\left(1-\cos ^{2} \theta\right)^{1 / 2}$, and $\Omega$ as in $R 1$.

J2. The density actually sampled in $R 3$ is $p(\rho, \theta) d \rho d \theta=\rho d \rho d \theta / \pi$, with marginal density $p_{1}(\rho) d \rho=2 p d \rho$. Under the latter, the density of $S=\rho^{2}$ is $q(S)=1$ on $(0,1)$. Hence, $S$ may be used in place of $r_{3}$ in $R 1$.

Note. If R3 of R3 is used, one avoids unnecessary square roots by setting

$$
\omega_{1}=x\left\{S^{-1}\left(1-\cos ^{2} \theta\right)\right\}^{1 / 2}, \omega_{2}=y\left\{S^{-1}\left(1-\cos ^{2} \theta\right)\right\}^{1 / 2}
$$

R5. Uniform direction $\Omega=\left(\omega_{7}, \ldots, \omega_{N}\right)$ in $N$-space.

B. If $S \equiv \sum_{1}^{N} v_{i}^{2}<1$, where $v_{i}=2 r_{i}-1$, set $\omega_{i}=v_{i} / S^{1 / 2}$.

Note. Efficiency $=\pi^{N / 2} / 2^{N-1} N \Gamma(N / 2)+0$, less than $1 / 2$ for $N>3$.

(FB). See C37 for al ternative.

R6. $p(x)=A^{-1} p_{1}(x)\left\{p_{2}(h(x))-P_{2}(g(x))\right\}:(a, b), p_{1}(x)$ density on $(a, b)$,

$P_{2}(x)$ density on $(c, d), P_{2}(y)=\int_{c}^{y} P_{2}(y) d y, c<g(x)<h(x)<d$. 
R. Sample $p_{1}(x)$ for $x$ on $(a, b)$, and $p_{2}(y)$ for $y$ on $(c, d)$.

Accept $x$ if $g(x)<y<h(x)$.

J. Since $\int_{g(x)<y<h(x)} p_{1}(x) d x p_{2}(y) d y=\int_{a}^{b} p_{1}(x) d x \int_{g(x)}^{h(x)} p_{2}(y) d y$

$=\int_{a}^{b} p_{1}(x)\left\{p_{2}(h(x))-p_{2}(g(x))\right\} d x=A, A$ is the probability of acceptance

(efficiency), and hence $p(x) d x$ is the probability of an accepted $x$ lying on $(x, x+d x)$.

R7. $p(x)=A^{-1} p_{1}(x) h(x) ;(a, b), p_{1}(x)$ density on $(a, b), 0<h(x)<1$.

B. Sample $p_{1}(x)$ for $x$ on $(a, b)$. Accept $x$ if $r_{1}<h(x)$.

J. Special case of $R 6$, with $c=0, d=1, p_{2}(y)=1, g(x)=0$. Efficiency $=$ A.

Note. The method is useful in Klein-Nishina and Thomson (incoherent and coherent) scattering modified by form factors. Due to the nature of the latter, efficiency considerations make it advisable to take the KleinNishina density for $p_{1}(x)$ and the form factor for $h(x)$ in incoherent scattering, whereas in coherent scattering, $p_{1}(x)$ is based on the form factor and $h(x)$ on the Thomson cross section. For details, see a forthcoming $L A$ report on the MCP code, which incorporates the method referred to.

R7A. $p(x) \equiv A^{-1} \cdot(b-a)^{-1} \cdot p(x) / M:(a, b), M=\max p(x), A=1 /(b-a) M$.

R. Accept $x \equiv a+r_{0}(b-a)$ if $r_{1}<p(x) / M$.

j. Special case of $R 7$, with $p_{1}(x)=1 /(b-a)$ uniform, and $h(x)=p(x) / M<1$. 
R7B. $p(x)=k^{-1} f(x) ;(a, b), k>0, k * \int_{a}^{b} f(x) d x$

R. Accept $x \equiv a+r_{0}(b-a)$ if $r_{1}<f(x) / D$, where $D=\max f(x)$.

J. Spectal case of R7A, with $h(x)=p(x) / M=k^{-1} f(x) / k^{-1} D=f(x) / D$.

Note. If $C \equiv \min f(x)$, then effictency $=$

$A=1 /(b-a) M=k /(b-a) D=\int_{a}^{b} f(x) d x /(b-a) D>\int_{a}^{b}\{f(x)-c\} d x /(b-a)(D-c)$.

R7C. $p(\phi)=k^{-1} f(\phi), f(\phi)=K-B \cos ^{2} \phi ;(0,2 \pi), K>B>0$.

R]. Accept $\phi \equiv 2 \pi r_{0}$ if $r_{1}<f(\phi) / K$.

J1. Special case of R7B. Effictency $>1 / 2$. (Note, R7B)

R2. Obtain $\cos \phi$ from R3 and accept if next $r<f(\phi) / K$.

Note. This density occurs in polarized Compton scattering, where $\phi$ itself is not required.

R7D. $\left.P(\phi)=k^{-1} f(\phi), f(\phi)=K-s^{2} i Q \cos 2 \phi+U \sin 2 \phi\right)$

$=K-H \cos 2\left(\phi-\phi_{0}\right) ;(0,2 \pi), H=S^{2}\left(Q^{2}+U^{2}\right)<K$,

$C \equiv \min f(\phi)=K-H, D \equiv \max f(\phi)=K+H \quad$.

R1. Accept $\phi=2 \pi r_{0}$ if $r_{1}<f(\phi) / D$.

J1. Special case of R7B. Efficiency $>1 / 2$ (Note, R7B)

R2. Obtain $\cos \phi, \sin \phi$ by R3; compute $\cos 2 \phi=\cos ^{2} \phi-\sin ^{2} \phi$, $\sin 2 \phi=2 \sin \phi \cos \phi$. Accept if next $r<f(\phi) / 0$. 
Note. The density occurs in polarized Compton scattering, where $\phi$ itself is not required.

R8. $p(v)=c_{1}^{-1} v^{n-1} /\left(\Lambda^{-1} e^{\nu}+1\right) ;(0, \infty), 0<\Lambda<1, n=3 / 2,2,5 / 2,3, \ldots$,

$c_{1}=\zeta_{a}(\Lambda, n) \Gamma(n), \quad \zeta_{a}(\Lambda, n) \equiv \sum_{1}^{\infty}(-1)^{j+1} \Lambda^{j} / j^{n} \quad$ (F11).

R. Sample $q(\nu)$ for $\nu$ on $(0, \infty)$ by $C 46$; accept $\nu$ if $r_{0}>\Lambda$, or if $\Lambda>r_{0}>\Lambda e^{-\nu}$.

J. One can write $p(\nu)=c^{-1} h(\nu) q(\nu)$, where $c^{-1}=\zeta_{u}(\Lambda, n) / \zeta_{a}(\Lambda, n)$. $\zeta_{u}(\Lambda, n) \equiv \sum_{1}^{\infty} \Lambda^{2 j-1} /(2 j-1)^{n}, 0<1-\Lambda<h(\nu) \equiv 1-\Lambda e^{-\nu}<1$, and $q(v)=v^{n-1} \Lambda e^{-v} /\left(1-\Lambda^{2} e^{-2 \nu}\right) \zeta_{u}(\Lambda, n) \Gamma(n)$ is the density of c46. The rule follows from $R$.

R9. $\bar{p}(x)=E_{b}^{-1} x^{n-1} e^{-b \sqrt{x^{2}+1}} ;(0, \infty), b>0, n=2,3,4, \ldots$,

$E_{b}=2^{\frac{n-1}{2}}(\Gamma(n / 2) / \Gamma(1 / 2)) k_{\frac{n+1}{2}}(b) / b^{\frac{n-1}{2}}$

B. Sample $q(v)=D_{b}^{-1} v^{n-1} e^{-b \nu}$ for $\nu$ on $(1, \infty)$ by $c 47$; accept $v$ with probability $h(\nu)=\left(1-1 / \nu^{2}\right)^{\frac{n}{2}-1}$. For accepted $\nu$, set $x=\left(\nu^{2}-1\right)^{1 / 2}$.

J. Under the transformation $x^{2}+1=v^{2}$, one has

$\bar{p}(x) d x=p(v) d v \equiv\left(E_{b}^{-1} D_{b}\right)\left(1-1 / v^{2}\right)^{\frac{n}{2}-1}\left(D_{b}^{-1} v^{n-1} e^{-b v} d v\right)$, and the rule follows from $R 7$. 
Note. For $n=2, h(v) \equiv 1$, acceptance is certain, and $D_{b}=E_{b}$. For $n=3, \bar{p}(x)$ is the Maxwell-Juttner relativistic momentum density [3] .

R10. $\bar{p}(x)=A^{-1} x^{n-1} /\left(\Lambda^{-1} e^{a \sqrt{x^{2}+1}}+1\right) ;(0, \infty), a>0,0<\Lambda<1, n=2,3,4, \ldots$ $A=2^{\frac{n-1}{2}}(\Gamma(n / 2) / \Gamma(1 / 2)) \sum_{1}^{\infty}(-1)^{j+1} \Lambda^{j} k_{\frac{n+1}{2}}(j a) /(j a)^{\frac{n-1}{2}}$

R. Sample $\bar{q}(v)$ for $v$ on $(1, \infty)$ by C48. Accept $v$ if $r_{0}<\left(1-1 / v^{2}\right)^{\frac{n}{2}-1}\left(1-\Lambda e^{-a \nu}\right)$. For accepted $\nu$, set $x=\left(\nu^{2}-1\right)^{1 / 2}$.

J. Under the transformation $x^{2}+1=v^{2}$, one has $\bar{p}(x) d x=p(v) d v$ $=D A^{-1} \cdot\left(1-1 / \nu^{2}\right)^{\frac{n}{2}-1}\left(1-\Lambda e^{-a v}\right) \bar{q}(v)$ for the $\bar{q}(v)$ in $C 48$, and the rule follows from R7.

R11. $\bar{p}(E)=c^{-1} e^{-a E} \sinh \sqrt{b E} ;(0, \infty), a, b>0$.

R. Define $K=1+(b / 8 a), L=a^{-1}\left\{K+\sqrt{K^{2}-1}\right\}, M=a L-1>0$.

Set $x=-\ln r_{1}, y=-\ln r_{2}$. Accept $x$ if $\{y-M(x+1)\}^{2}<b L x$. For accepted $x_{3}$ set $E=L x$.

J. The rule follows from $R 6$ and $C 17$. Let $E=L x$ for arbitrary $L>0$. Then $\bar{p}(E) d E=p(x) d x \equiv \frac{1}{2} c^{-1} L e^{a L-1} \cdot e^{-x} \cdot e^{-(a L-1)(x+1)}\left\{e^{\sqrt{L L x}}-e^{-\sqrt{\sigma L x}}\right\} d x$ $=\frac{1}{2} c^{-1} L e^{M} \cdot e^{-x} \cdot\left\{e^{-g(x)}-e^{-h(x)}\right\}=A^{-1} p_{1}(x)\left\{P_{2}(h(x))-P_{2}(g(x))\right\} d x$, where $M=a L-1, g(x)=M(x+1)-\sqrt{b L x}, h(x)=M(x+1)+\sqrt{6 L x}$, $p_{1}(x)=e^{-x}, p_{2}(y)=e^{-y}, p_{2}(y)=\int_{0}^{y} p_{2}(y) d y$. The above choice of $L$ insures that $g(x)>0$ on $(0, \infty)$. For a check, let $a=3, b=6$. (After Kalos [10]) 
R12. $p(x)=\sqrt{2 / \pi} e^{-x^{2} / 2} ;(0, \infty)$

R. Set $x=-\ln r_{1}, y=-\ln r_{2}$. Accept $x$ if $(x-1)^{2}<2 y$.

J. Special case of $R 6$, with $a=0=c, b=\infty=d, p_{1}(x)=e^{-x}$,

$p_{2}(y)=e^{-y}, P_{2}(y)=1-e^{-y}, g(x)=(x-1)^{2} / 2, h(x) \equiv \infty$. Efficiency $A=(\pi / 2 e)^{1 / 2}=.76$.

Note. For $\bar{p}(x)=(2 \pi)^{-1 / 2} e^{-x^{2} / 2}$ on $(-\infty, \infty)$ choose $( \pm)$ sign for accepted $x$ above with probability $1 / 2$.

R13. $p\left(\frac{\alpha^{-}}{\alpha}, \theta\right)$; Polarized Compton scattering.

R. A method of sampling the Klein-Nishina cross section for polarized photons is given in [4]. This involves $C 61$ and $R 7 C, D$. The fit used for $C 61$ (cf. $[5,6]$ ) is an improvement on that given in [4] .

\section{Note on Statistics}

In judging the reliability of a sampling procedure, the following test may be usefur. For a discrete density, pre-compute $p_{i}=p(i)$ for any desired set of argument values $v=i$. For a continuous density, compute $\int_{a_{i}}^{b_{i}} p(v) d v=p_{i}$ for a suitable set of intervals $\left(a_{i}, b_{i}\right)$. Let the density $p(v)$ be sampled $N$ times, according to the rule given, and tally the number $f_{j}$ of times the sample $v=i$, or $v \varepsilon\left(a_{i}, b_{i}\right)$. Fixing attention on any one index $i$, this yields a Bernoulli sequence of $N$ trials, with $f_{i}$ the number of "successes", and $p_{i}$ the probability of success, $q_{i}=1-p_{i}$ the probability of "failure", in any one sampling.

In this situation, the law of large numbers states that $P_{N} \equiv P\left\{\left|f_{i} / N-P_{j}\right|<\rho p_{j}\right\}>1-\left(q_{j} / \rho^{2} p_{j} N\right) \rightarrow 1$ while the central limit theorem asserts that $P_{N}=\phi\left(\rho \sqrt{p_{i} N / q_{i}}\right)+z_{N}$, where $\phi(z)=\frac{2}{\sqrt{2 \pi}} \int_{0}^{z} e^{-z^{2} / 2} d z$, and $z_{N}+0$. 
One may note that, insofar as a method is correct, its statistical reliability depends only on the density itself.

\section{REFERENCES}

1. Ki. M. Case, F. de Hoffmann, G. Placzek, Introduction to the Theory of Neutron Diffusion, Vol. I (Los Alamos Scientific Laboratory, 1953) p. 153.

2. E. D. Cashwe11, C. J. Everett, G. D. Turner, "A Method of Sampling Certain Probability Densities Without Inversion of Their Distribution Functions," Los Alamos Scientific Laboratory report LAMS (in preparation).

3. S. Chandrasekhar, An Introduction to the Study of Stellar Structure (Dover Pubilications, Inc., 1958) Ch. X.

4. C. J. Everett, "A Relativity Notebook for Monte Carlo Practice," Los Alamos Scientific Laboratory report LA-3839 (1968).

5. C. J. Everett, E. D. Cashwell, "Approximation for the Inverse of the KleinNishina Probability Distribution," Los Alamos Scientific Laboratory report LA-4448 (1970).

6. C. J. Everett, E. D. Cashwe11, G. D. Turner, "A New Method of Sampling the Klein-Nishina Probability Distribution for All Incident Photon Energies Above $1 \mathrm{keV}, "$ Los Alamos Scientific Laboratory report LA-4663 (1971).

7. C. J. Everett, P. R. Stein, "On Random Sequences of Integers," Bull. Amer. Math. Soc., 76, 349-351 (1970).

8. C. J. Everett, P. R. Stein, "On Random Sequences of Integers," Los Alamos Scientific Laboratory report LA-4268 (1969).

9. H. Kahn, "Applications of Monte Car10," Rand Corporation report RM-1237-AEC (1956).

10. M. H. Kalos, F. R. Nakache, J. Celnik, Monte Carlo Methods in Reactor Computations, Computing Methods in Reactor Physics (Gordon and Breach, N. Y., 1968) Ch. 5.

11. M. H. Kalos, "Monte Carlo Cälculations of the Ground State of Three- and Four-Body Nuclei," Phys. Rev. 1282, 1791-1795 (1962).

i2. G. N. Watson, A Treatise on the Theory of Bessel Functions (Cambridge Univ. Press, 1952) pp. 183, 185. 